Une idée graphique du costume : les maquettes du cinéma français de l'après-guerre dans les collections de la Cinémathèque française

A graphic idea of costume: the post-war french cinema sketches of the Cinémathèque française collection

\title{
Myriam Fouillet
}

\section{OpenEdition}

Journals

Édition électronique

URL : https://journals.openedition.org/itti/1489

DOI : $10.4000 /$ itti. 1489

Éditeur

Université de Poitiers

Référence électronique

Myriam Fouillet, « Une idée graphique du costume : les maquettes du cinéma français de l'aprèsguerre dans les collections de la Cinémathèque française », Images du travail, travail des images [En ligne], 10 | 2021, mis en ligne le 20 février 2021, consulté le 25 janvier 2022. URL : http:// journals.openedition.org/itti/1489; DOI : https://doi.org/10.4000/itti.1489

Ce document a été généré automatiquement le 25 janvier 2022.

Images du travail, travail des images 


\title{
Une idée graphique du costume : les maquettes du cinéma français de l'après-guerre dans les collections de la Cinémathèque française
}

\author{
A graphic idea of costume: the post-war french cinema sketches of the \\ Cinémathèque française collection
}

Myriam Fouillet

Je remercie le personnel de la Cinémathèque française pour son aide, particulièrement Jacques Ayroles, Françoise Lémerige, Joël Daire et Sandra Laupa.

1 Une maquette de costume est une œuvre graphique, souvent accompagnée d'échantillons de tissus et d'annotations, ayant pour but de représenter en deux dimensions un projet de costume afin de procéder à son exécution en trois dimensions. L'utilisation de ce type de dessins pour le cinéma trouve son origine dans l'usage déjà bien ancré de maquettes de costumes dans les arts de la scène, mais les contraintes de leur conception sont différentes, car la vision du costume au cinéma est médiatisée ${ }^{1}$ et possiblement rapprochée, alors qu'elle est directe et lointaine au théâtre, à l'opéra ou en danse. La Cinémathèque française conserve cent quatre-vingt-treize maquettes de costumes peintes ou dessinées issues du travail de huit dessinateurs pour la préparation de vingt-cinq longs métrages de fiction produits en France entre 1945 et 1959.

2 Ce corpus exclut un ensemble de dessins de La Reine Margot (Jean Dréville, 1954) inventoriés comme maquettes de costumes, mais en fait réalisés par le décorateur Alexandre Hinkis pendant le tournage, en témoignage de la vie sur le plateau. Les dessins de préparation de Mon oncle (Jacques Tati, 1956) par Pierre Étaix n'ont pas été intégrés, non plus, à mon étude, car cet ensemble complexe à décrypter nécessite une analyse à part entière ${ }^{2}$. Ces cas révèlent les problèmes soulevés par la qualification d'un dessin en maquette, l'attribution à un auteur ou l'identification d'un film. Un costume 
de "page de Cendrillon» dessiné par Monique Plotin est présumé avoir été dessiné pour le cinéma, mais, sans preuve, il a été écarté. Enfin, le corpus ne prendra pas en compte deux maquettes de costumes réalisées par Alexandre Trauner pour le projet Hécatombe, d'après un scénario de Jacques Prévert, car le film n'a jamais été produit.

Mon matériau comprend dix-neuf films historiques, deux se déroulant dans un univers merveilleux et un dans un pays non occidental. Trois, seulement, ont une intrigue contemporaine. La très forte proportion de maquettes de costumes historiques et merveilleux peut s'expliquer soit parce qu'ils ont été conservés justement pour leur nature jugée plus remarquable, soit parce que dessiner des costumes n'aurait pas toujours été nécessaire pour une production avec des vêtements contemporains. De même, on trouve treize films en couleurs, alors que la production était en grande majorité en noir et blanc, et ainsi une trop large part de films à budget élevé. Ce corpus n'est pas représentatif de toute la production d'après-guerre, car il est aussi lié à la décision subjective des créateurs de conserver ces documents de travail et aux dons aléatoires et rares qu'a pu recevoir la Cinémathèque française. Ainsi, certains films sont très bien représentés, comme Les Grandes Manœeuvres (René Clair, 1955, Eastmancolor), avec un ensemble de cinquante-et-un dessins qui, figurant une large galerie de personnages et de scènes, donne une idée de l'envergure du travail de conception des costumes pour une seule production. Au contraire, la Cinémathèque française conserve une seule maquette pour certains films, à l'image de Lucrèce Borgia (Christian-Jaque, 1952, Technicolor). Cependant, même les grands ensembles ne semblent pas complets. En effet, la robe blanche à pois noirs portée par Michèle Morgan pour interpréter Marie-Louise dans Les Grandes Manœuvres est absente.

L'intérêt de ce matériau se trouve notamment dans la diversité des dessinateurs, avec de grands noms très actifs pendant la période étudiée, comme Georges Annenkov, Rosine Delamare ou Marcel Escoffier, mais aussi des créateurs moins connus, telle Blanche Van Parys. Quelques maquettes témoignent même du travail sur les costumes d'artistes, célèbres chefs décorateurs, comme Georges Wakhévitch, ou peintres, comme Mayo ou Georges Koskas. Cependant, leur œuvre est encore peu étudiée, ces créateurs restant des figures de l'ombre du monde cinématographique (même si elles sont plus connues que les petites mains comme les habilleuses). Malgré le fait qu'elles apparaissaient parfois dans les articles de la presse spécialisée de l'époque, n'étant pas systématiquement citées aux génériques et rarement sur le matériel publicitaire, elles étaient généralement moins valorisées que les personnalités d'autres métiers comme celui de scénariste.

5 Nonobstant certains manques, les maquettes étudiées témoignent du processus de création des costumes des films français d'après-guerre. Une analyse détaillée de ces vestiges nous renseigne sur leur place dans la chaîne opératoire. Les maquettes de costumes sont des œuvres graphiques originales produites par l'imagination et le talent d'artistes-techniciens. Mais la destination première des dessins est de rendre visibles les idées vestimentaires imaginées par le créateur afin que les costumes soient exécutés et s'intègrent pleinement à une œuvre cinématographique singulière. Ainsi, ces outils servant de médiateurs dans un travail collectif sont augmentés d'échantillons et d'inscriptions pour une interprétation plus fidèle. À travers ces différentes fonctions des maquettes de costumes, nous verrons que ces dernières rendent compte des habitudes professionnelles de l'époque ainsi que de la progression de la pensée créative 
et permettent de mieux cerner le rôle des créateurs dans l'élaboration et la réalisation d'un costume.

\section{Le style personnel des créateurs de costumes}

Ce qui frappe d'abord l'observateur, c'est la dimension esthétique des maquettes de costumes. Celle-ci varie en fonction des créateurs. Se pose alors la question des habitudes graphiques et des méthodes de travail de chacun d'eux. Comparer les maquettes permet de distinguer des styles vestimentaires, comme on le ferait avec l'œuvre de grands couturiers.

7 Georges Annenkov ${ }^{3}$ utilise une grande diversité de formats de papier, allant de $25 \times 11,5 \mathrm{~cm}$ à $64 \times 29 \mathrm{~cm}$, mais le format qu'il utilise le plus est $50 \times 35 \mathrm{~cm}$. Il dessine à la mine de graphite, repasse certains contours à l'encre et colore ses figures à l'aquarelle ou à la gouache. Le fond est généralement laissé sans ornement, mais il arrive qu'Annenkov trace un encadré, une ligne de sol, telle qu'on peut en voir sur la maquette du costume $\mathrm{n}^{\circ} 1$ de Louis $\mathrm{I}^{\mathrm{er}}$ de Bavière (D104-060) pour Lola Montès (Max Ophuls, 1955, Eastmancolor), ou un décor esquissé rapidement, comme un pâturage en montagne pour La Symphonie pastorale (Jean Delannoy, 1946, noir et blanc) (D094-002). Les visages de ses personnages sont souvent des portraits des acteurs. On reconnait Danielle Darrieux sur le dessin du costume $\mathrm{n}^{\circ} 2$ de Mme de Rênal (D104-030 $)$ pour Le Rouge et le Noir (Claude Autant-Lara, 1954, Eastmancolor). S'il ne connaît pas l'identité des futurs interprètes ou ne souhaite pas prendre le temps de personnaliser ses maquettes, Annenkov figure les personnages sans visage, notamment pour la robe $n^{\circ} 3$ de Mme de Rênal (D104-032), ou avec un visage flou, que l'on observe sur le dessin du costume $\mathrm{n}^{\circ} 7$ de Julien Sorel (D104-028). Il exagère la finesse de la taille féminine. Ses traits sont délicats. Il représente les costumes avec précision, en dessine chaque élément, par exemple de fins galons autour des volants du corsage de la tenue $\mathrm{n}^{\circ} 2$ de Mme de Rênal (D104-0305) ou des perles ornant les manches de Rafaella (D104-052) dans Patrie (Louis Daquin, 1945, noir et blanc). Cependant, ses dessins peuvent être parfois plus synthétiques, à l'image de ceux réalisés pour les classes populaires de Patrie. Pour les personnages du cirque de Lola Montès, son style est plus géométrique et audacieux, prenant ses distances avec les formes vestimentaires du milieu du XIX ${ }^{e}$ siècle, comme sur la maquette D003-096 ${ }^{6}$. Ces divergences s'expliquent par sa conception du métier de créateur de costumes qui «trichera volontairement dans la technique de ses dessins, il trahira son coup de crayon, sa manière artistique et prendra le chemin le plus facile ${ }^{7}$ " afin de plaire au réalisateur, au producteur et aux acteurs. Ses maquettes comportent des échantillons. Sa signature peut prendre plusieurs formes : soit son nom entier, soit ses initiales, soit l'initiale de son prénom suivie de son nom de famille. 
Illustration 1 : Georges Annenkov, Le Rouge et le Noir : Madame de Rênal n2, 1954, mine de graphite, gouache, encre sur papier, $50 \times 35 \mathrm{~cm}$.

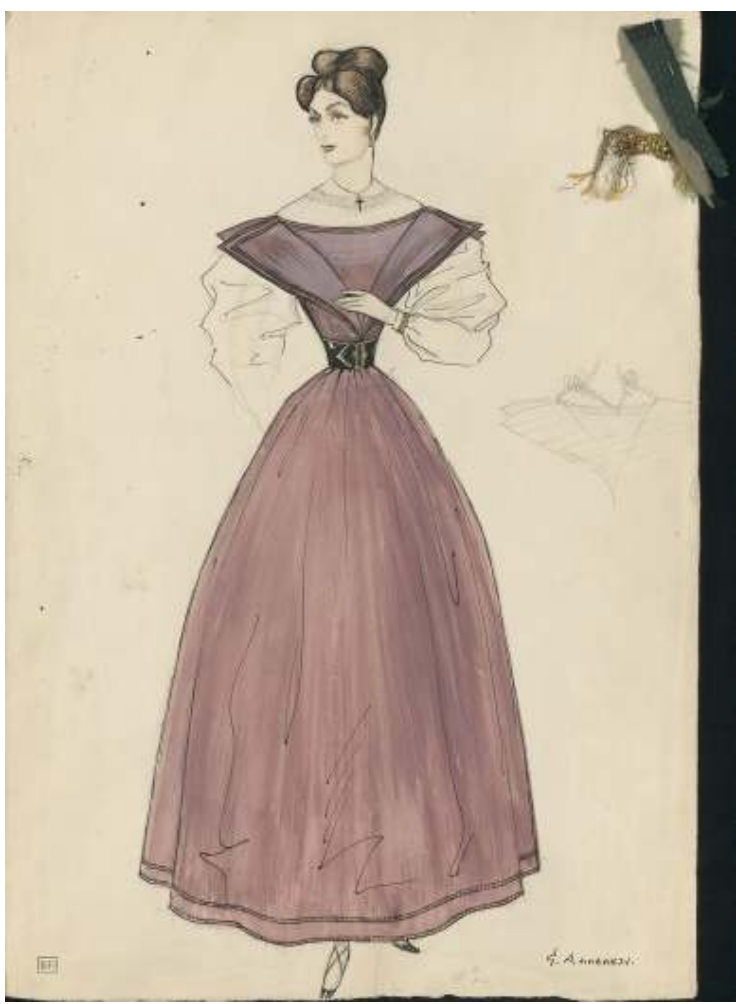

(c) Dominique Rabusson, coll. Cinémathèque française, Paris, D104-030. 
Illustration 2 : Georges Annenkov, Lola Montès : Cirque, 1955, encre, lavis d'encre, gouache sur carton, $35,5 \times 21,5 \mathrm{~cm}$.

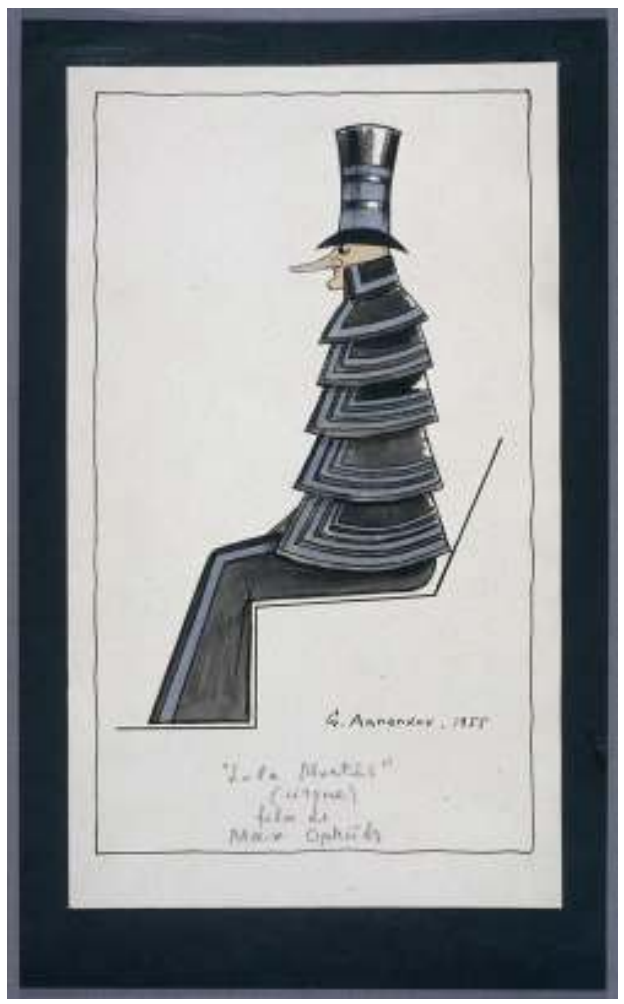

(c) Dominique Rabusson, coll. Cinémathèque française, Paris, D003-096.

Rosine Delamare ${ }^{8}$ utilise en grande majorité des feuilles aux formats proches du demiraisin, $50 \times 32,5 \mathrm{~cm}$. Elle se sert beaucoup de gouache mais rarement seule. Elle la marie avec la mine de graphite ou l'encre comme avec la maquette de Nèle (D024-030) pour Les Aventures de Till l'Espiègle (Gérard Philipe, 1956, Technicolor). Elle utilise aussi souvent l'aquarelle, parfois l'aquarelle et la gouache mêlées, parfois le feutre comme sur la maquette du "salon Duverger » (D025-091) des Grandes Manœuvres (René Clair, 1955). Elle ne cherche pas à occuper toute la page, habituellement le fond reste neutre. Les personnages n'occupent parfois que la moitié de la page, comme Titine (D019-058) dans French Cancan (Jean Renoir, 1954, Technicolor). Ils sont souvent représentés en action : patinant sur la glace, le vent dans les jupes, pour Les Aventures de Till l'Espiègle (D024-030) ou dansant dans les bras d'un homme évoqué en quelques traits pour le dessin D025-090 des Grandes Manœuvres. Les silhouettes féminines de Rosine Delamare ont la taille fine et les hanches développées, comme on peut le voir avec le personnage de Marguerite (D024-065) pour Marguerite de la nuit (Claude Autant-Lara, 1955, Technicolor). Son coup de crayon est souple, sûr et rapide, tout en courbes et contrecourbes. Ses costumes sont dessinés soit avec un trait fin, comme le montre le dessin de Marguerite de la nuit (D024-065), soit avec un pinceau plus chargé, tel qu'on l'observe sur la maquette de Porte des Lilas (René Clair, 1956, noir et blanc) (D024-066). Rosine Delamare semble apprécier particulièrement les ensembles. Elle fait partie de ceux qui utilisent le plus les échantillons sur les maquettes, de dentelle en particulier.

Marcel Escoffier ${ }^{9}$ dessine sur des feuilles de différents formats, dont un presque carré de 25,5 × 24,5 cm pour Lucrèce Borgia (D102-054), mais semble apprécier le format demiraisin. On peut lire en relief dans le papier d'une maquette de La Belle Otero (Richard 
Pottier, 1954, Eastmancolor) (D102-041) le nom de la marque Arches France, maison auprès de laquelle il se fournissait et qui est reconnue pour sa qualité. Il dessine aussi sur bloc-notes comme le montre le feuillet représentant un déshabillé pour Le Secret de Mayerling (Jean Delannoy, 1948, noir et blanc) (D104-018). Il privilégie presque toujours la mine de graphite, et la gouache pour la mise en couleurs. Il use très souvent de l'aquarelle, parfois du feutre et de l'encre, mais rarement d'une technique peu répandue, le crayon de couleur, qu'il a utilisée pour la maquette de Maria Vetsera pendant la partie de chasse du Secret de Mayerling (D102-030). Disposés sur un fond neutre, les personnages peuvent occuper la page entière comme moins de sa moitié. Les traits de Marcel Escoffier sont d'une très grande finesse. Chaque élément est minutieusement et très proprement légendé, avec des flèches comme un schéma de sciences naturelles. Les indications sont nombreuses: on en trouve douze pour le costume $\mathrm{n}^{\circ} 1$ de Mme Hédouin (D102-035), directrice du magasin dans Pot-Bouille (Julien Duvivier, 1957, noir et blanc). Pour la tenue de La Belle Otero (D102-041), il précise: " plumes noires » et « fond de soie " pour le chapeau ; « orchidée posée hors du bras » pour les manches et «biais de velours » pour les ornements du corsage qui est composé de "chantilly noir sur satin ». Cependant, il utilise peu les échantillons. Une seule maquette à la Cinémathèque française en comporte, celle du costume $\mathrm{n}^{\circ} 1$ de la baronne Vetsera pour Le Secret de Mayerling (D102-021). On peut supposer qu'il préfère indiquer par écrit les matériaux utilisés. Notons que certains de ses dessins définitifs ne sont pas colorés. Il signe très souvent simplement de son prénom, « Marcel ».

10 Pour préparer Goha (Jacques Baratier, 1957, Agfacolor), Georges Koskas ${ }^{10}$ utilise le format $38,70 \times 27,90 \mathrm{~cm}$. Ses personnages et costumes sont évoqués par des traits épais réalisés en quelques coups de pinceau chargé de gouache sur un fond aquarellé. Il signe de son nom précédé de l'initiale de son prénom.

11 Antoine Mayo ${ }^{11}$ travaille sur des feuilles de différents formats allant de $40 \times 25 \mathrm{~cm}$ à $55 \times$ $36 \mathrm{~cm}$, mais semble apprécier le $49 \times 30 \mathrm{~cm}$. Il n'utilise pas systématiquement la mine graphite, mais souvent la gouache et l'aquarelle, et parfois un lavis d'encre. Ses pages sont pleinement occupées, l'arrière-plan est saturé : d'autres personnages entourent la figure principale, comme un photographe et un cycliste (D015-017) pour Trois femmes (André Michel, 1951, noir et blanc), ou des décors qui les contextualisent, tels des arcades et un dôme (D068-079) pour La Beauté du diable (René Clair, 1949, noir et blanc) ou les falaises des côtes normandes (D015-022) pour Une vie (Alexandre Astruc, 1957, Eastmancolor). Les personnages occupent presque toute la hauteur de la page, comme sur la maquette de Casque d'Or (Jacques Becker, 1951, noir et blanc) (D014-098). De plus, leurs corps antinaturalistes ont des proportions exagérées : de longs cous, des visages allongés et des traits synthétiques. Parfois, ces personnages sont si évanescents qu'ils sont dénués de visage, comme dans Une vie (D015-019), ou que leurs membres sont à peine mentionnés, comme on peut le constater sur le dessin D015-018 pour Une vie. Le rapide coup de pinceau d'Antoine Mayo crée un effet de flou. Il ne s'attarde pas sur des précisions, il travaille par petites touches pour évoquer des détails, comme une dentelle bordant un col de chemise de nuit dans Une vie (D015-021). Mais ce n'est pas systématique, car pour Le Rideau cramoisi (Alexandre Astruc, 1952, noir et blanc), le dessin est plus classique, plus précis (D015-03012), et, pour La Beauté du diable (D014-097), il sculpte les ombres et lumières. Les maquettes conservées à la Cinémathèque française ne comportent aucun échantillon de tissu. Peut-être Antoine Mayo n'avait-il pas 
l'habitude d'attacher les textiles choisis aux maquettes correspondantes. Sa signature est bien lisible, mais il ajoutait peu d'indications écrites.

Illustration 3 : Antoine Mayo, Le Rideau cramoisi, 1952, mine de graphite, aquarelle sur papier, $40 \times 25 \mathrm{~cm}$.

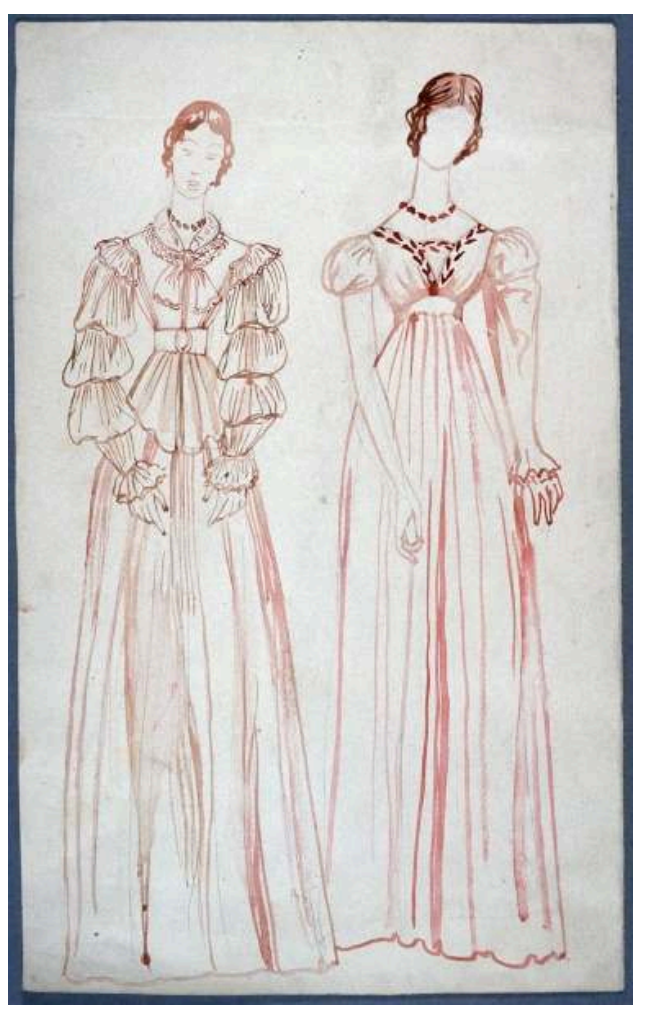

Cinémathèque française, Paris, D015-030, (c) ADAGP.

Pour L'Amour, Madame (Gilles Grangier, 1951, noir et blanc), sur un fond restant vide, Blanche Van Parys ${ }^{13}$ dessine ses costumes à la mine de graphite, les colore à la gouache et leur donne plus de relief en représentant des reflets de lumière avec des rehauts blancs (D020-094). Avec des traits légers et aériens, elle figure des personnages minces, élancés, aux visages délicats, présentés de face, sans accessoires, mais avec une attitude discrète, comme des mannequins qui défilent dans un salon élégant. Elle n'a pas épinglé d'échantillons de tissu, mais donne des indications par écrit et signe ses œuvres.

Les maquettes de Georges Wakhevitch ${ }^{14}$ ont été réalisées à la gouache, principalement sur des feuilles au format $56 \times 37 \mathrm{~cm}$. Les personnages, disposés sur un fond blanc, prennent des attitudes particulières et sont accompagnés de quelques accessoires ou petits éléments de décors, comme l'arbalète de Pascal ou le marteau, l'enclume et la souche d'arbre de Giglio (D084-063) dans Barbe-Bleue (Christian-Jaque, 1951, Gevacolor). On peut remarquer la grande précision des traits de Wakhevitch qui trace des contours bien nets. Il a dessiné les coutures latérales de la robe d'Aline pour Barbe-Bleue (D021-087) ou encore les fins ornements de ses manches, les motifs des tissus. Sa méticulosité permet de distinguer le relief et la technique de la broderie à l'or sur la hanche gauche du personnage de Barbe-Bleue (D021-088). Ses coups de pinceau ne sont pas visibles, car il réalise des aplats de couleur très uniformes. Il n'ajoute aucun échantillon de tissu mais signe son œuvre. 
14 Ainsi, malgré l'apparition à plusieurs reprises du demi-raisin, la diversité des formats de papier montre que ces créateurs ne sont pas attachés au respect des normes françaises des beaux-arts et adaptent sans contrainte les dimensions à leurs besoins. De la même manière, les dessinateurs usent librement de techniques variées, mais, cette fois, les procédés traditionnels prédominent. De plus, la fréquence des signatures montre une volonté de marquer comme travaux finis des dessins que les techniciens estiment réussis ou de se revendiquer comme auteurs de ce qu'ils considèrent comme une œuvre d'art à part entière. Les signatures ne sont pas toujours présentes, peut-être parce que ces dessins sont perçus par leurs créateurs comme des éléments intermédiaires d'un travail au long court. À la Cinémathèque française, les maquettes sont collectées pour leurs valeurs esthétiques et documentaires. C'est aussi pour ces raisons qu'elles sont encadrées, mises en lumière et présentées sur des cartels dans des expositions où souvent le public d'aujourd'hui les apprécie comme des œuvres des beaux-arts, pour leur beauté, indépendamment des films, participant ainsi à la reconnaissance de leurs artistes.

\section{Des œuvres pratiques tournées vers une concrétisation}

Cependant, d'après Georges Annenkov, « dans le cinéma, seul l'effet obtenu à l'écran est l'œuvre d'art, tandis que la maquette n'est que l'un des sentiers qui mènent vers le but final ${ }^{15}$ ». À l'image d'autres domaines des arts décoratifs, ces dessins n'ont pas qu'une fonction esthétique, leur finalité pratique est également importante. Les maquettes portent les traces d'une phase de préparation. Elles prennent rarement la forme de portraits. Ainsi, l'ensemble de L'Affaire du collier de la reine (Marcel L'Herbier, 1945, noir et blanc) de Georges Annenkov représentant le bailli de Suffren, Cagliostro et le cardinal de Rohan (D003-069 ${ }^{16}$ ) parait déroutant au premier abord. Si l'on compare ces dessins à des portraits anciens de ces hommes célèbres, notamment une gravure du cardinal de Rohan par Jeune Voyes ${ }^{17}$ ou des bustes sculptés de Cagliostro ${ }^{18}$ et Pierre André de Suffren ${ }^{19}$ par Jean-Antoine Houdon, on découvre qu'Annenkov les a soigneusement copiés, peut-être pour des recherches documentant la période qu'il devait représenter. Annenkov cherche aussi de l'inspiration dans l'art pictural. Pour une étude de costume d'enfant noble dans Patrie (D104-053 ${ }^{20}$ ), il s'est inspiré d'un portrait d'Antoine van Dyck représentant Guillaume II, prince d'Orange, et sa fiancée Marie Stuart ${ }^{21}$ : il en reprend la position, mais simplifie la tenue avec un col et des rubans plus sobres, l'absence de cape, de gants, de broderies et de crevées. Dans leurs maquettes, les créateurs de costumes peuvent aborder de différentes manières les œuvres dont les films sont des adaptations. Pour le spectacle mythologique de Nana (Christian-Jaque, 1954, Eastmancolor), Marcel Escoffier n'a conservé de la description d'Émile Zola que les longs cheveux blonds et le fait que l'héroïne soit couverte de gros bijoux d'or (D102-040 et D102-037). Alors qu'au contraire, avec le dessin D102-035, représentant le premier costume de Madame Hédouin dans Pot-Bouille, il est plutôt fidèle aux mots de l'auteur en choisissant une robe gris-noir, un col blanc et une petite cravate d'homme. 
Illustration 4 : Georges Annenkov, L'Affaire du collier de la reine, 1945, mine de graphite sur papier et carton, $39 \times 87,5 \mathrm{~cm}$.

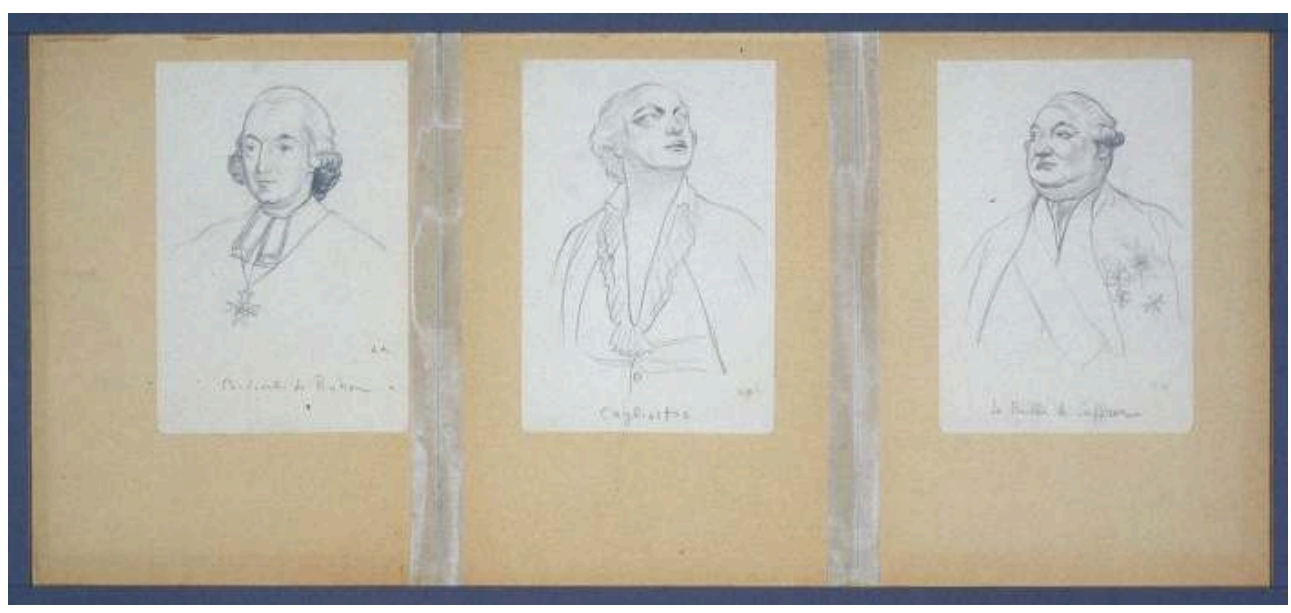

(c) Dominique Rabusson, coll. Cinémathèque française, Paris, D003-069.

Illustration 5 : Jeune Voyes, Louis René Édouard Prince de Rohan, Guémené, Cardinal de la Sainte Église Romaine, Évêque Prince de Strasbourg, 1892, gravure, $43,5 \times 31,5 \mathrm{~cm}$.

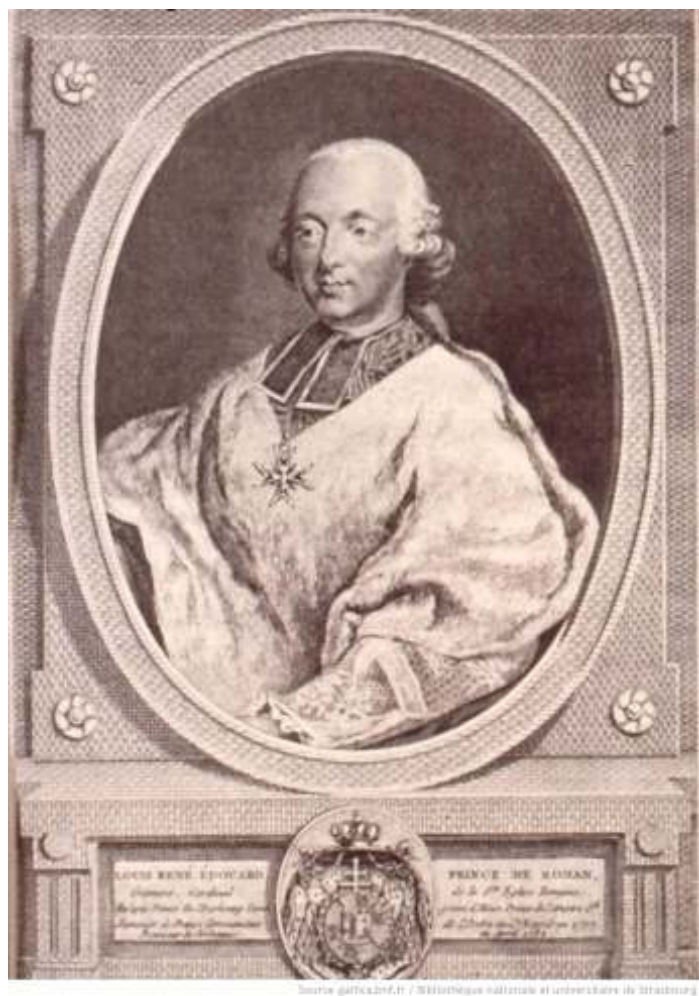

Bibliothèque nationale et universitaire de Strasbourg, Strasbourg, NIM29762. 
Illustration 6 : Jean Antoine Houdon, Giuseppe Balsamo, comte de Cagliostro, 1786, marbre, $62,9 \times 58,9 \times 34,3 \mathrm{~cm}$.

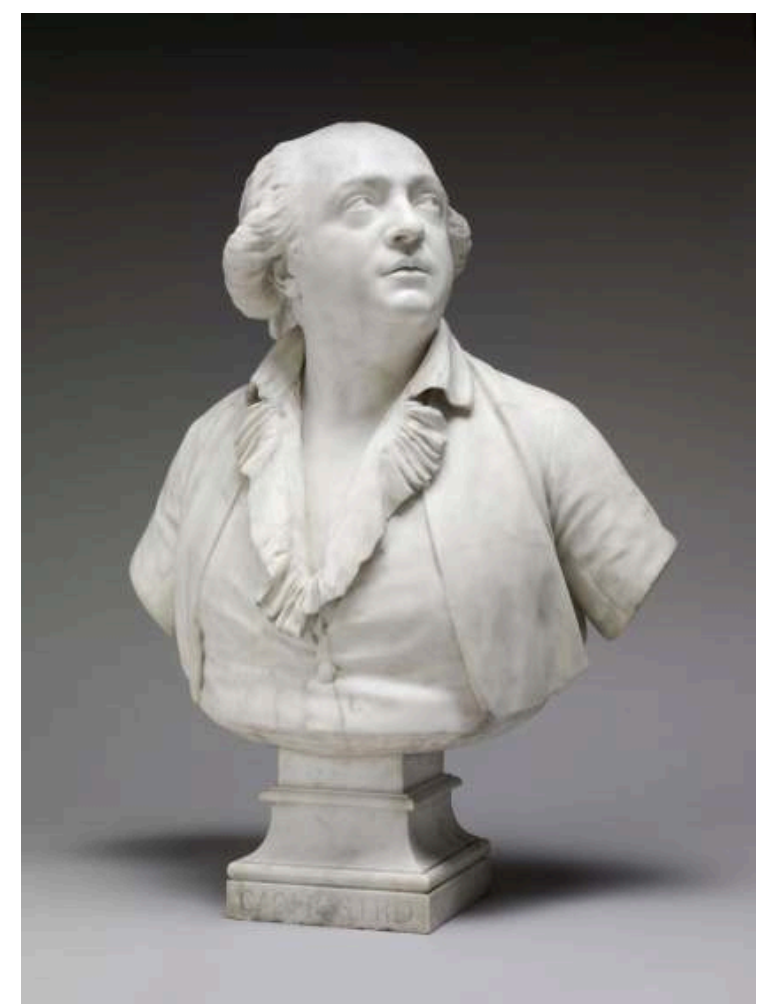

Samuel H. Kress Collection, National Gallery of Art, Washington D.C., 1952.5.103. 
Illustration 7 : Jean Antoine Houdon, Pierre-André de Suffren, 1786-1787, marbre, $179 \times 73 \times 73 \mathrm{~cm}$.

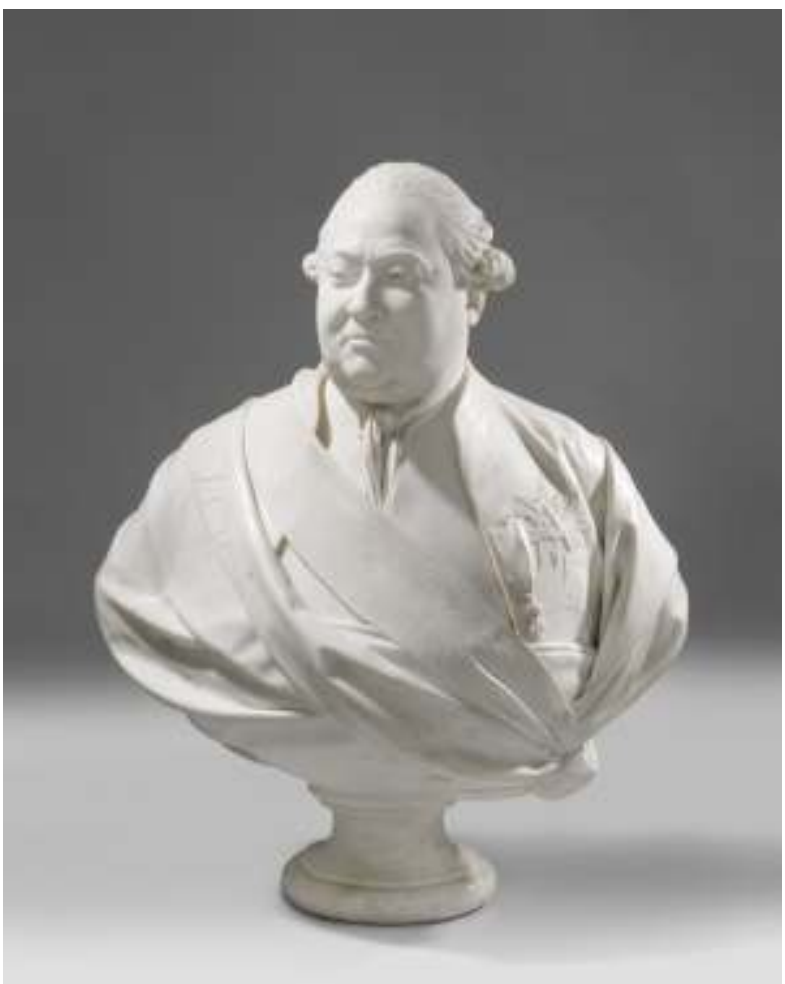

Cabinet royal de peinture des Pays-Bas, Rijksmuseum, Amsterdam, BK-C-2003-3, http:// hdl.handle.net/10934/RM0001.COLLECT.377343. 
Illustration 8 : Georges Annenkov, Patrie : Étude de costume - enfant noble, 1945, mine de graphite, gouache sur papier, $48 \times 32 \mathrm{~cm}$.

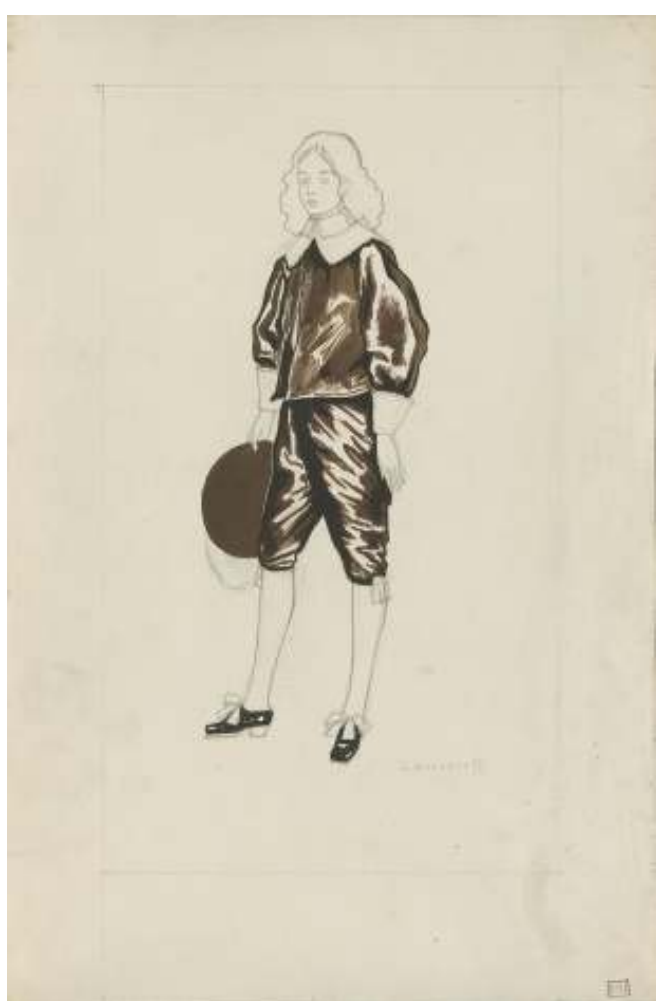

(c) Dominique Rabusson, coll. Cinémathèque française, Paris, D104-053. 
Illustration 9 : Antoine Van Dyck, Guillaume II, prince d'Orange et sa fiancée Marie Stuart, 1641, huile sur toile, $182,5 \times 142 \mathrm{~cm}$.

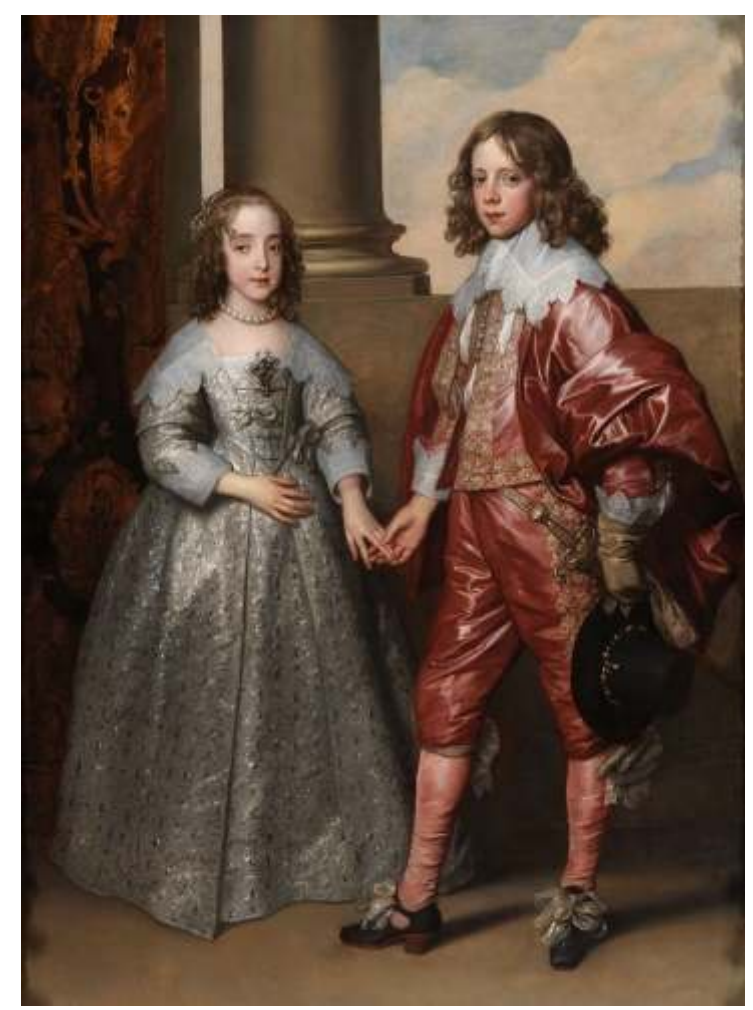

Rijksmuseum, Amsterdam, SK-A-102.

Les maquettes sont au centre de la conception des costumes. En effet, les idées posées sur le papier deviennent plus concrètes et aident les créateurs dans leur réflexion. Certains dessins semblent être des ébauches, notamment ceux sans couleur, réalisés au crayon gris avec des traits fins par Annenkov. Des études comparatives confirment que certains créateurs dessinent une esquisse préparatoire avant de réaliser une maquette définitive. Le personnage et les formes du costume du dessin D102-02422 pour Le Secret de Mayerling, exécuté par Escoffier avec un coup de crayon très léger, se retrouvent sur le dessin D102-023 $3^{23}$, représentant le costume $\mathrm{n}^{\circ} 6$ de la comtesse Larisch, lorsqu'elle rend visite à Rudolf au palais, réalisé avec un coup de crayon plus appuyé, plus sûr et des formes plus précises, plus détaillées. D'autres dessins commencés avec une intention plus ferme n'ont manifestement pas été terminés. Par exemple, pour le costume de Marie Vestera à cheval dans Le Secret de Mayerling (D102-017), les contours généraux sont tracés de manière sûre au crayon gris, mais les détails ne sont pas dessinés, ni le visage du personnage qui n'est pas mis en couleurs. De plus, contrairement aux habitudes d'Escoffier, le schéma n'est pas légendé. On peut ainsi imaginer que l'auteur a finalement abandonné une idée qui était pourtant avancée. Le repentir est plus évident lorsqu'une figure a été barrée, comme sur un dessin représentant Nini dans French Cancan (D025-037). Le fait que certains éléments ne sont pas mis en couleurs suggérerait que finalement cette pièce de vêtement ne sera pas exécutée, comme c'est le cas pour la cape esquissée sur les épaules du costume » n11 » de Julien Sorel pour Le Rouge et le Noir (D104-026). 
Illustration 10 : Marcel Escoffier, Le Secret de Mayerling, 1948, mine de graphite sur papier, $50 \times 32,5 \mathrm{~cm}$.

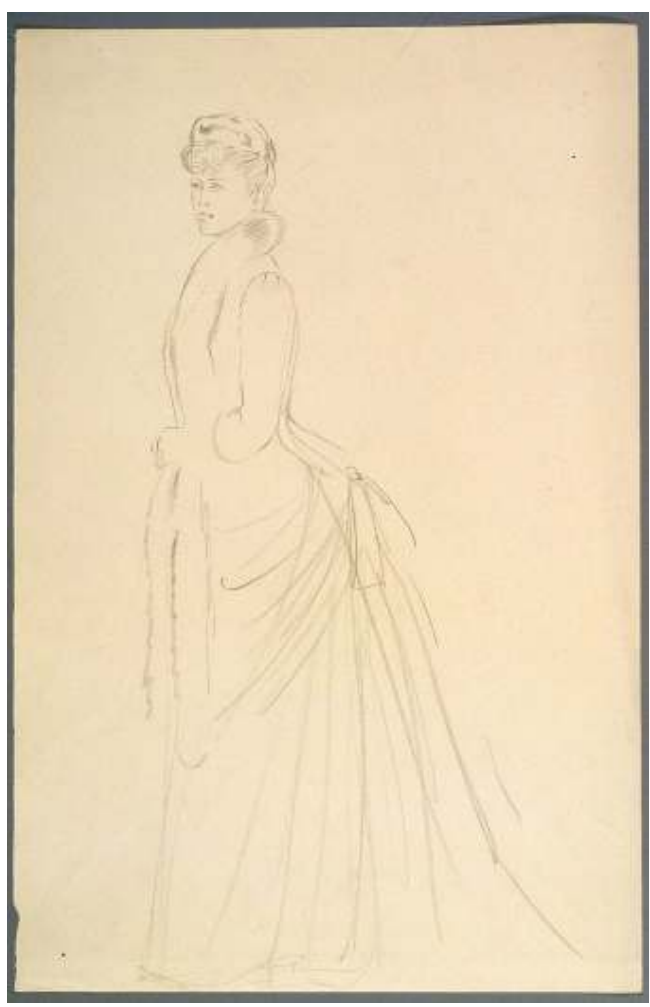

(c) Carol Escoffier, coll. Cinémathèque française, Paris, D102-024 
Illustration 11 : Marcel Escoffier, Le Secret de Mayerling, 1948, mine de graphite sur papier, $50 \times 32,5 \mathrm{~cm}$.

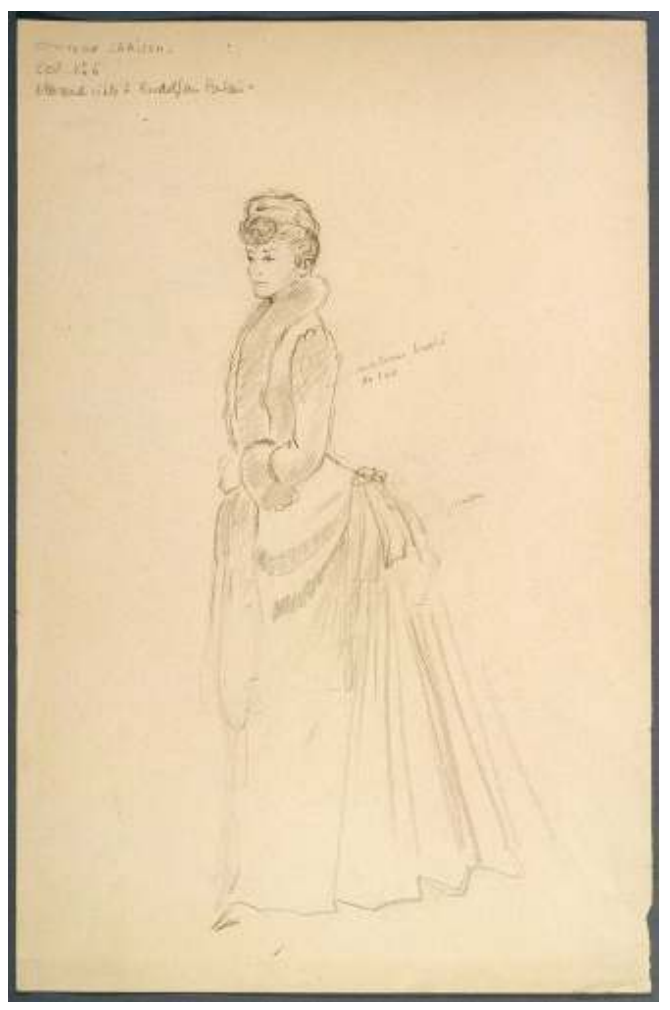

(c) Carol Escoffier, coll. Cinémathèque française, Paris, D102-023.

Les maquettes témoignent de la pensée du costume en versions multiples. À côté d'un dessin principal, certains maquettistes dessinent une variante d'une partie du costume. Sur le dessin D104-030 ${ }^{24}$, Annenkov a représenté une variation du corsage de Mme de Rênal : les volants obliques sont un peu plus larges et le col de la chemise est godronné et prend la forme d'un $\mathrm{V}$ alors que celui du dessin principal est arrondi et resserré en fronces. Pour Les Grandes Mancuvres, le dessin D026-00125 représente Marie-Louise Rivière dans son magasin de modiste, portant un corsage au col relevé et ouvert, aux manches trois quarts, orné de nombreux volants couvrant les épaules, la poitrine et les bras. En haut à gauche de la page, le corsage est plus sobre, orné de bandes verticales, avec un col fermé aux revers arrondis et des manches longues aux poignets serrés. Rosine Delamare a finalement opté pour la seconde option mais avec une ceinture droite $^{26}$. On peut trouver deux variations de costumes pour un même personnage sur une même page. Pour Le Rideau cramoisi (D015-03027), le bras droit du personnage de droite est nu, simplement surmonté d'une manche ballon, alors que son bras gauche est recouvert d'une manche longue. Cela témoigne de la comparaison de deux essais distincts de la part du concepteur, Mayo. La Cinémathèque française possède deux versions d'une tenue pour une même scène : le costume de la colonelle pour le bal de la Croix-Rouge des Grandes Manœuvres. Elles sont similaires par la coiffure, les lunettes, le collier, les boucles d'oreille, les gants, la dentelle au bord du décolleté, la forme générale de la robe, mais on peut observer quelques différences : le dessin D025-098 ${ }^{28}$ présente une tenue dont le châle est posé sur les bras, avec un pouf sur les fesses, alors que le dessin D025-097 ${ }^{29}$ présente une robe avec trois rangées de volants supplémentaires, et le châle est attaché au centre du corsage, dissimulant ce dernier. 
De même, on trouve deux maquettes très similaires pour L'Affaire du collier de la reine. Elles présentent le même personnage, une noble dame de la cour, dans la même position avec quasiment la même tenue: le dessin D003-068 ${ }^{30}$ présente une robe maintenue sur les côtés de la jupe grâce à des nœuds de rubans bleu clair, comme celui que l'on trouve au milieu du corsage, et un chapeau rouge avec un ruban bleu, tandis que sur le dessin D003-066 ${ }^{31}$, le chapeau est rose saumon pâle, le corsage possède des manches et des engageantes, la taille est nouée d'un long ruban bleu et la robe est ouverte en triangle sur la jupe qui est ornée d'un plus grand nombre de volants. Ces deux exemples témoignent d'une hésitation entre deux possibilités créatives très abouties. Une telle précision des couleurs, notamment pour le chapeau, concernant un film en noir et blanc n'a rien d'étonnant, car la variété des nuances de gris obtenues par des costumes en couleurs permettait de construire la lisibilité de l'image, en élaborant une distinction entre les tons de gris des différents costumes et les tons de gris des éléments de décor qui se détachaient ainsi les uns des autres à l'écran.

Illustration 12 : Rosine Delamare, Les Grandes Manœuvres, 1955, mine de graphite, aquarelle gouache sur papier, $50 \times 32 \mathrm{~cm}$.

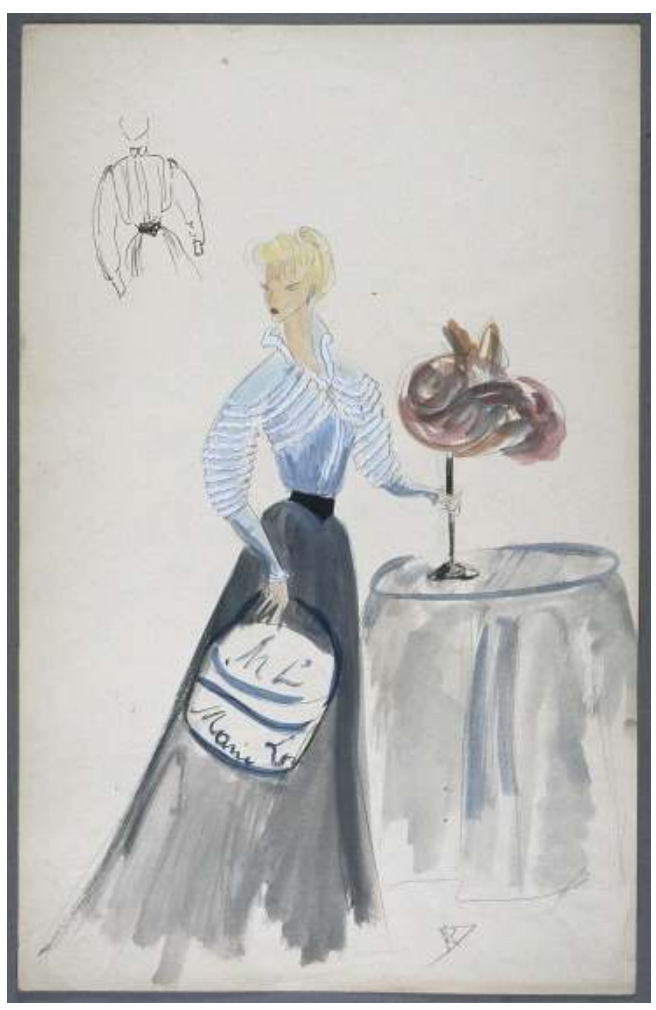

(c) Anne-Marie Spitzer, coll. Cinémathèque française, Paris, D026-001. 
Illustration 13 : Marie-Louise Rivière, photogramme des Grandes Manœuvres.

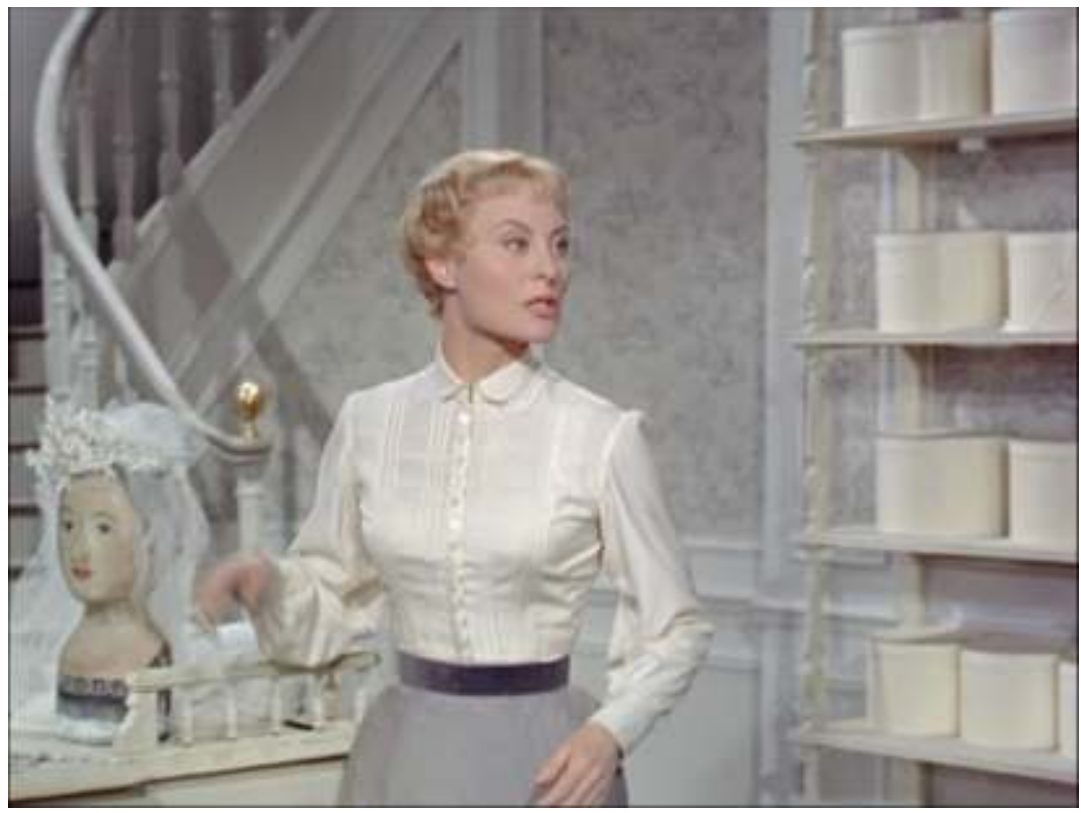

Illustration 14 : Rosine Delamare, Les Grandes Manœuvres : la colonelle, 1955, aquarelle, gouache sur papier, $50 \times 32 \mathrm{~cm}$.

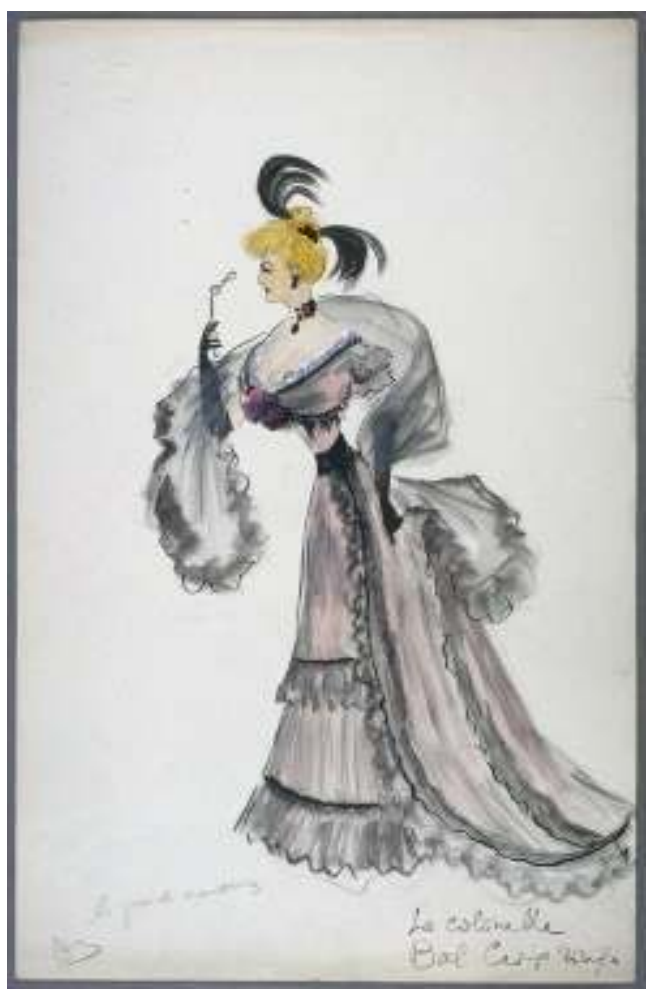

(c) Anne-Marie Spitzer, coll. Cinémathèque française, Paris, D025-098. 
Illustration 15 : Rosine Delamare, Les Grandes Manœuvres : la colonelle, 1955, aquarelle, gouache sur papier, $50 \times 32 \mathrm{~cm}$.

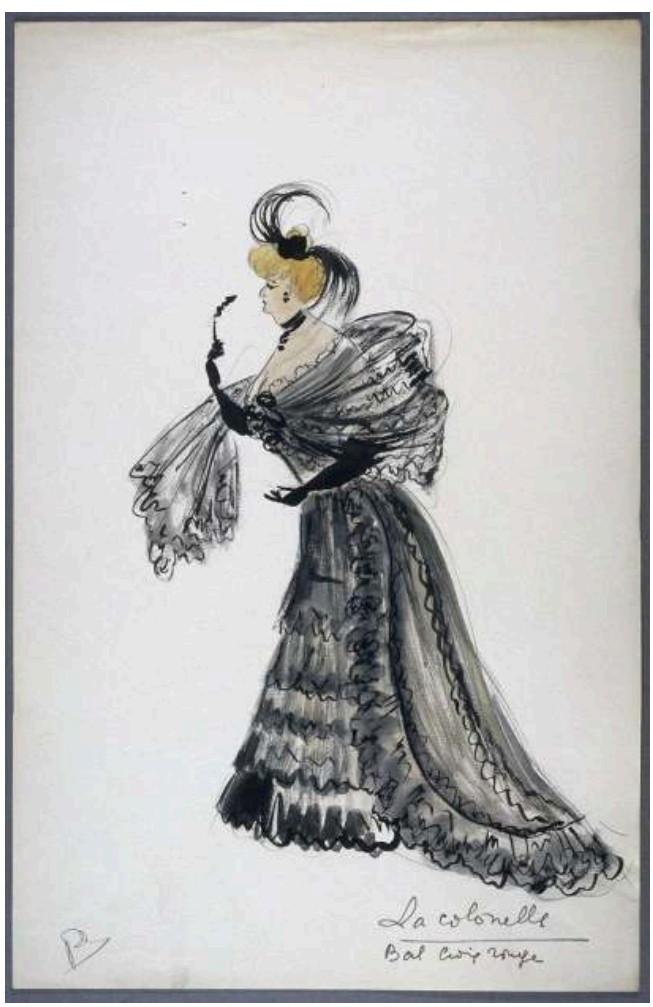

(c) Anne-Marie Spitzer, coll. Cinémathèque française, Paris, D025-097.

Illustration 16 : Georges Annenkov, L'Affaire du collier de la reine, 1945, mine de graphite, aquarelle sur papier, $33 \times 50 \mathrm{~cm}$.

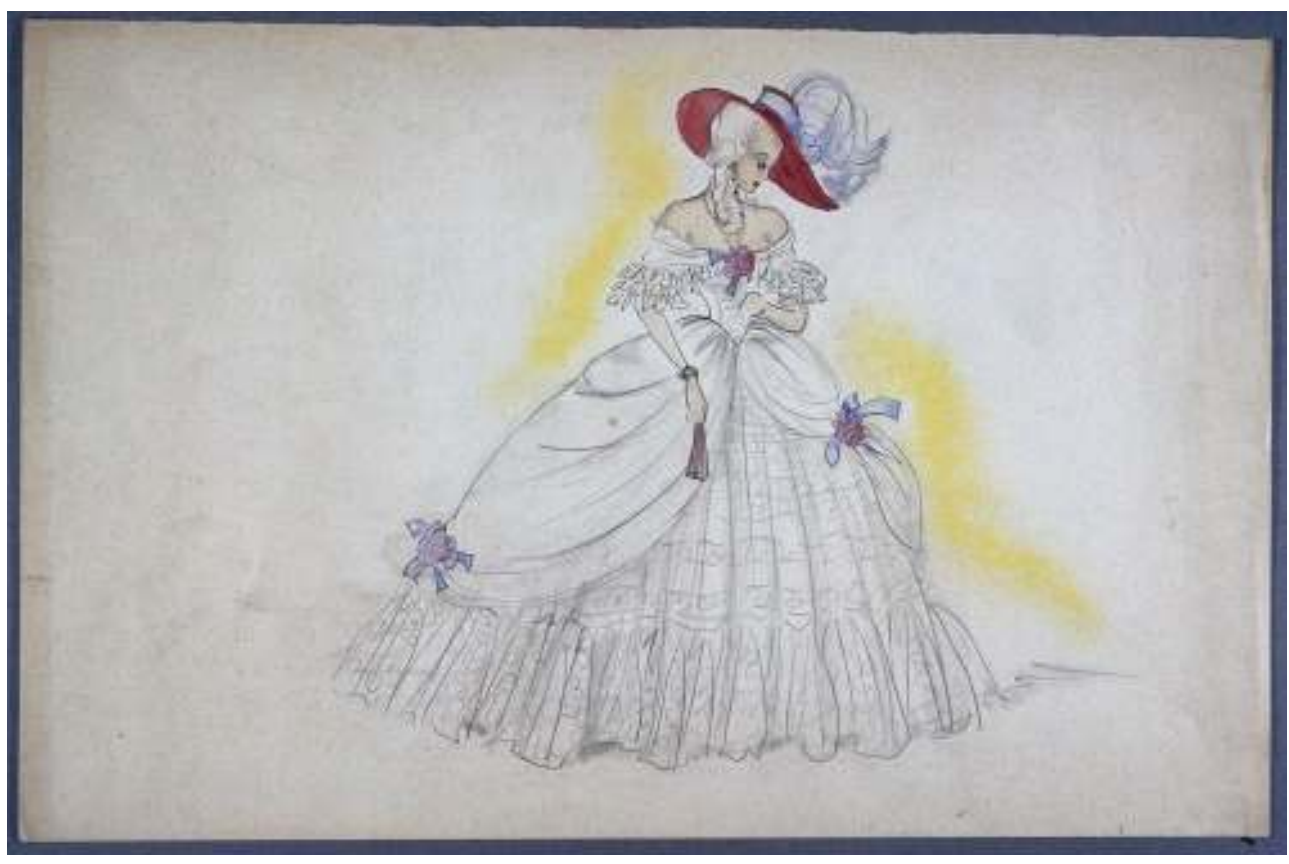

(c) Dominique Rabusson, coll. Cinémathèque française, Paris, D003-068. 
Illustration 17 : Georges Annenkov, L'Affaire du collier de la reine, 1945, mine de graphite, aquarelle sur papier, $32 \times 49,5 \mathrm{~cm}$.

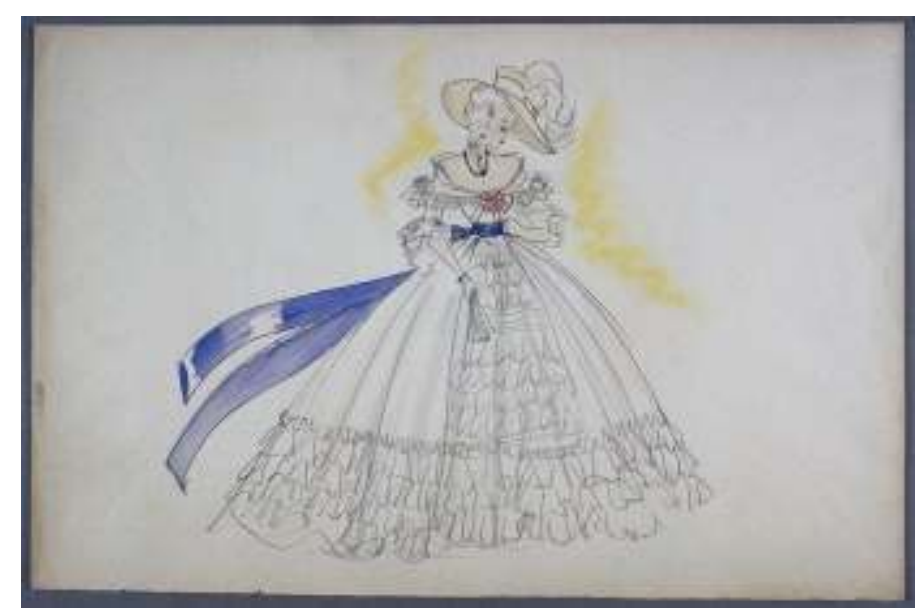

(c) Dominique Rabusson, coll. Cinémathèque française, Paris, D003-066.

18 Les maquettes révèlent les habitudes de travail du métier, notamment concernant la représentation des personnages. Les costumes sont souvent tracés individuellement sur une feuille orientée en portrait. Plus rarement, ils sont dessinés sur une feuille orientée en paysage lorsque les personnages sont allongés, comme la Belle Abbesse dans French Cancan (D025-020), ou lorsque le costume est aussi large que haut, comme celui de Nana (D102-037). Les personnages sont majoritairement représentés en pied, souvent de face ou de trois quarts, mais plus rarement de profil comme Gertrude dans La Symphonie pastorale (D094-002), et encore plus rarement de dos, comme Juliette (D082-075), présentée de trois quarts dos, pour Juliette ou la Clé des songes (Marcel Carné, 1950, noir et blanc). Souvent, les personnages figurés de dos sont féminins et portent des robes aux volumes très développés à l'arrière ou très longues, formant traîne. Sur le dessin de French Cancan D025-00932, Rosine Delamare a représenté de dos un manteau du soir pour aller à la Reine Blanche, ce qui permet de voir les fronces en haut du dos et de comprendre d'où vient le gonflant à l'arrière et son ampleur. Les personnages sont souvent tracés dans une pose relativement neutre, mais mettant en valeur la nature vivante des étoffes qui composent leurs vêtements. L'attitude nonchalante visible sur un dessin d'Une vie (D015-023), un coude appuyé contre un arbre et une main posée sur la taille, rappelle les portraits de la noblesse $d u X^{X V I I}{ }^{e}$ siècle. Ces postures sont souvent déterminées par l'adjonction d'un accessoire, telle une Bible tenue à deux mains dans une position réservée pour Une vie (D015-019). Parfois les figures sont dessinées en action, comme le personnage de Pascal qui bande son arbalète dans Barbe-Bleue (D084-063). 
Illustration 18 : Rosine Delamare, French Cancan : Éleanor, 1954, mine de graphite, aquarelle sur papier, $50 \times 33 \mathrm{~cm}$.

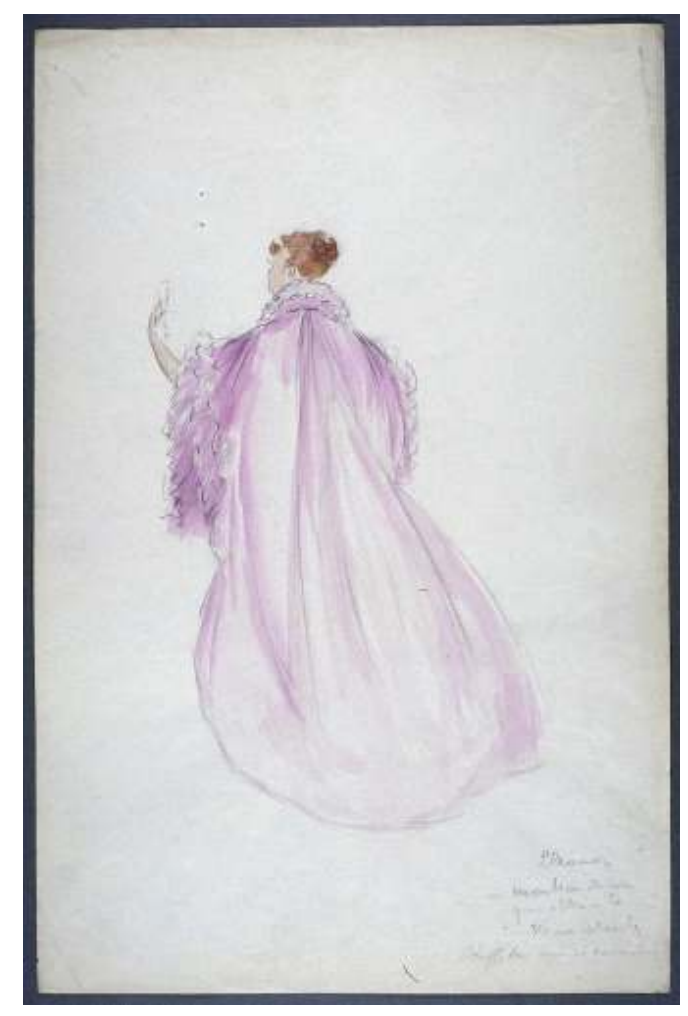

(c) Anne-Marie Spitzer, coll. Cinémathèque française, Paris, D025-009.

Une proportion plus modeste de maquettes regroupe plusieurs costumes sur un seul feuillet de manière à créer un ensemble. On découvre rarement deux costumes d'un même personnage sur une page, comme c'est le cas avec un dessin de Lola Montès (D104-058) qui présente, à droite, le costume $\mathrm{n}^{\circ} 3$ du roi et, à gauche, son costume $\mathrm{n}^{\circ} 2$. Certains créateurs, comme Annenkov, réalisent des montages de leurs dessins : deux ou trois dessins de costumes individuels sont rassemblés pour en former un seul présentant ainsi un groupe. C'est le cas pour trois personnages non identifiés de Patrie (D104-056) qui forment un groupe de bourgeois. Pour l'ensemble présentant deux hommes d'armes (D104-055 $5^{33}$ ), les deux parties sont harmonisées, unifiées, par le prolongement de l'escalier de la page de droite sur la page de gauche, et le découpage du contour du costume de l'officier espagnol chevauchant celui de Karloo, interprété par Jean Desailly. Il existe aussi des ensembles déjà constitués. Par exemple, le dessin « Mme de Rênal - M. de Rênal » (D024-067) de Rosine Delamare présente sept tenues de Louise, trois tenues de $\mathrm{M}$. de Rênal et d'autres personnages de la ville de province où ils demeurent, tel un paysan. Les costumes sont grossièrement esquissés de manière à en donner une idée globale, comme un résumé de leurs formes, de leurs couleurs et des silhouettes obtenues. Peut-être Rosine Delamare s'en est-elle servie pour comparer les costumes de personnages se côtoyant à l'image et contrôler l'harmonie des formes et des couleurs. Pour les uniformes des officiers espagnols des Aventures de Till l'Espiègle (D024-033), l'effet des couleurs entre elles est marquant, avec un fort contraste entre celles des armoiries espagnoles, le jaune et le rouge, et le noir typique des costumes espagnols du XVI ${ }^{e}$ siècle. On peut observer simplement deux personnages sur une page, comme les sœurs Duverger (D026-00734) qui, dans Les Grandes Manœuvres, apparaissent 
toujours conjointement. Il semble donc approprié de concevoir leurs costumes de concert. Rosine Delamare teste aussi les ensembles dans leur contexte, dans le décor correspondant. Sur « le salon Duverger " (D025-091), on reconnait les deux robes des sœurs, visibles sur le dessin D026-007 $7^{35}$, mises en scène avec leur ameublement. Sur les maquettes, on ne trouve pas uniquement les personnages principaux ou secondaires, mais aussi les figurants qui sont souvent décrits en groupe, ou du moins à plusieurs, comme le montre le dessin «Les figurants » d'Annenkov pour Patrie (D104-057). Rosine Delamare avait pour habitude de commencer la phase graphique par la représentation d'ensembles de figurants pour déterminer l'ambiance qui entourait les personnages principaux $^{36}$. On trouve aussi, parmi les ensembles, des groupes de personnages qui partagent une activité. Ils sont généralement moins nombreux et ainsi dépeints plus en détail, par exemple le "public du Bruant» (D025-042) ou le "public de la Reine Blanche" (D025-035) de French Cancan. Pour le même film, "Les blanchisseuses" (D084-029) et les danseuses du «studio de Guibole» (D025-021) sont représentées en action avec, fait surprenant, des échantillons de tissu, et une femme "chez Guibole » est accompagnée de mensurations (D025-005). Cela montre que, pour Rosine Delamare, les dessins d'ensembles sont de vrais outils de travail final.

Illustration 19 : Georges Annenkov, Patrie : Jean Desailly [Karloo] et un officier espagnol, 1945, mine de graphite, encre, gouache sur papier, $43 \times 34,5 \mathrm{~cm}$.

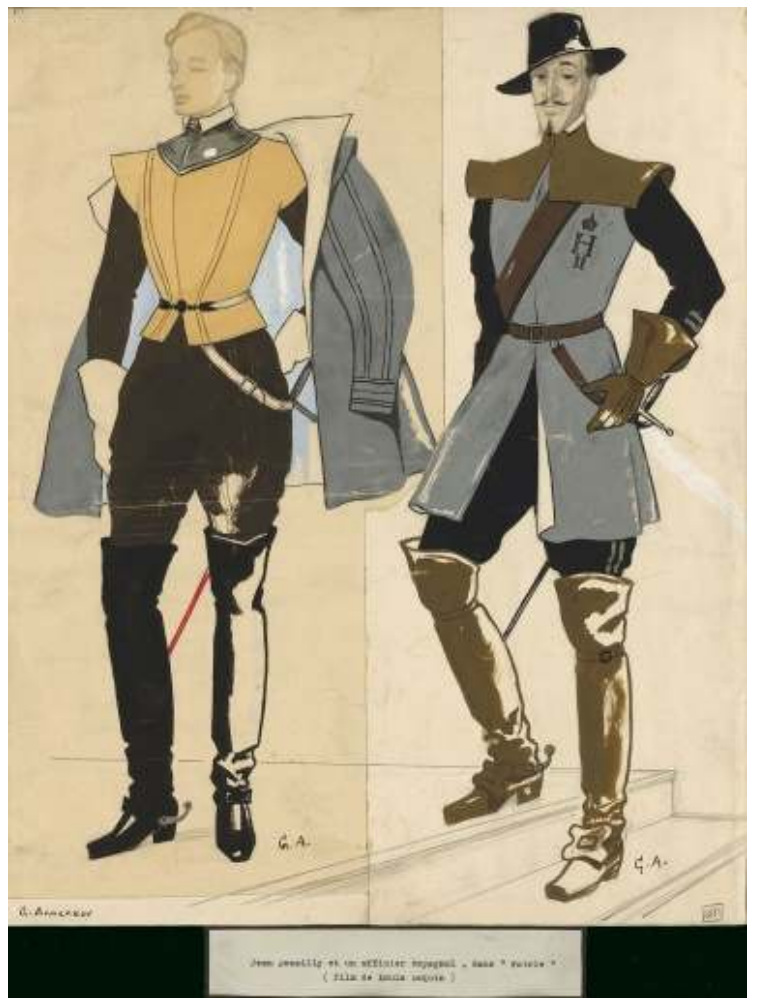

(c) Dominique Rabusson, coll. Cinémathèque française, Paris, D104-055. 
Illustration 20 : Rosine Delamare, Les Grandes Manœuvres : les sœurs Duverger reçoivent M. L., 1955, aquarelle, gouache sur papier, $50 \times 32 \mathrm{~cm}$.

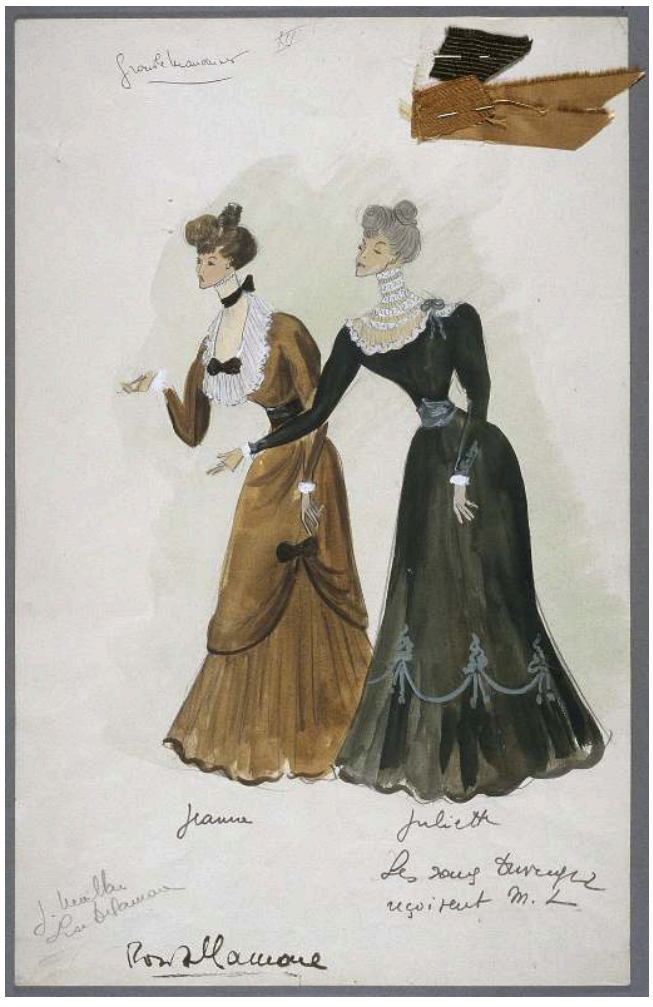

(c) Anne-Marie Spitzer, coll. Cinémathèque française, Paris, D026-007.

La clarté d'évocation des tenues prévaut sur l'agrément. Le créateur de costumes doit donc penser à préciser des détails. Il doit figurer l'état du costume, son tombé et la manière dont il est porté. Par exemple, pour l'homme à la pelle de Patrie (D104-046), Annenkov a dessiné des déchirures et un lacet dénoué. Le costume n'est pas un paramètre cinématographique fixe, il peut être modifié à l'image en cours de film. Lorsqu'un costume est modulable, c'est-à-dire que le personnage peut ajouter ou retirer certaines pièces de vêtement, les dessinateurs représentent souvent ces pièces sur une même feuille. Ainsi, Blanche Van Parys, pour L'Amour, Madame, évoque une femme portant des escarpins, une jupe droite avec une marinière en jersey, à gauche, et cette même tenue surmontée d'une veste à double boutonnage, à droite (D020-095). Pour la maquette du costume $\mathrm{n}^{\circ} 4 \mathrm{du}$ roi dans Lola Montès (D104-059 ${ }^{37}$ ), sur la droite, Annenkov a dessiné la veste qui est cachée par le manteau sur le dessin principal. Sur la droite de la planche décrivant le duc d'Albe pour Patrie (D104-050 38 ), il a dessiné la tête du même personnage surmontée d'un chapeau à plume. Rosine Delamare trace le jupon de Nini, à côté de la figure en chemise de corps, corset, culotte et bas, dans le dessin la dépeignant se rhabillant dans le fournil de French Cancan (D025-038 ${ }^{39}$ ). Le rôle du créateur de costumes est de concevoir une tenue entière. Ainsi, la plupart des maquettistes précisent dans leur dessin l'apparence des accessoires et des coiffures. On distingue sur le costume de Michèle Morgan dans La Symphonie pastorale de solides chaussures de montagne à larges talons avec des lacets surmontés d'une lanière attachée grâce à un fermoir à boucle. Autour du dessin principal, on peut trouver d'autres dessins, le plus souvent réalisés à la mine de graphite, précisant l'aspect de certains détails et représentés au même niveau. À gauche du couvre-chef du costume 
$\mathrm{n}^{\circ} 11$ de Julien Sorel dans Le Rouge et le Noir (D104-026), Annenkov a dessiné son képi de profil, ce qui permet de mieux distinguer sa forme; et à droite de son buste, la cordelette tressée à pompons est représentée en agrandissement. Dans ses écrits, Annenkov pense que le dessinateur doit faire « de nombreux croquis supplémentaires : détails des broderies, de dentelles, des plis, de la coupe ${ }^{40}$ ». La coiffe dite "French hood " d'Élisabeth dans Patrie (D104-051 ${ }^{41}$ ) est figurée de profil à droite du dessin principal. Blanche Van Parys, pour L'Amour, Madame, modélise un chapeau (D020-095) dans deux orientations différentes: à droite, vu de face, et à gauche, vu de côté. Parfois, les créateurs préfèrent réaliser des maquettes de détails. On trouve ainsi dans les collections de la Cinémathèque des maquettes d'accessoires, comme la D003-06 ${ }^{42}$ qui présente un soulier, un éventail, un binocle et une mule. On distingue alors clairement l'apparence de chaque objet: la mule est bordée de fronces, le soulier est recouvert d'un tissu fleuri, orné de boutons, de dentelle et d'une boucle métallique ouvragée. Le créateur de costumes prend donc en charge la conception d'une partie des accessoires. La limite entre sa fonction et celle de l'accessoiriste semble se situer dans la distinction entre ce qui est porté par les acteurs et ce qui est déposé dans le décor. La Cinémathèque française possède aussi des maquettes de coiffures, comme la D026-006 $6^{43}$ pour Les Grandes Manœuvres. Cette réflexion de Rosine Delamare sur les coiffures montre que c'est à elle qu'incombe la décision du style des coiffures ou, du moins, des chapeaux qui les surmontent. Enfin, il existe des maquettes de sousvêtements. Les jupons $\mathrm{du} \mathrm{XIX}^{\mathrm{e}}$ siècle, en particulier ceux portés pendant la III ${ }^{e}$ République, sont si complexes qu'ils nécessitent un dessin pour en clarifier les formes, comme le montre l'image D102-032 ${ }^{44}$ du Secret de Mayerling qui précise chaque fronce, chaque bouton, chaque couture. Pour plus de lisibilité, le créateur n'a pas dessiné de corps féminin occupant ces vêtements, mais leur volume correspond à celui qu'ils prennent lorsqu'ils sont portés. 
Illustration 21 : Georges Annenkov, Lola Montès : König n4, 1955, mine de graphite sur papier, encre, gouache, $55 \times 29 \mathrm{~cm}$.

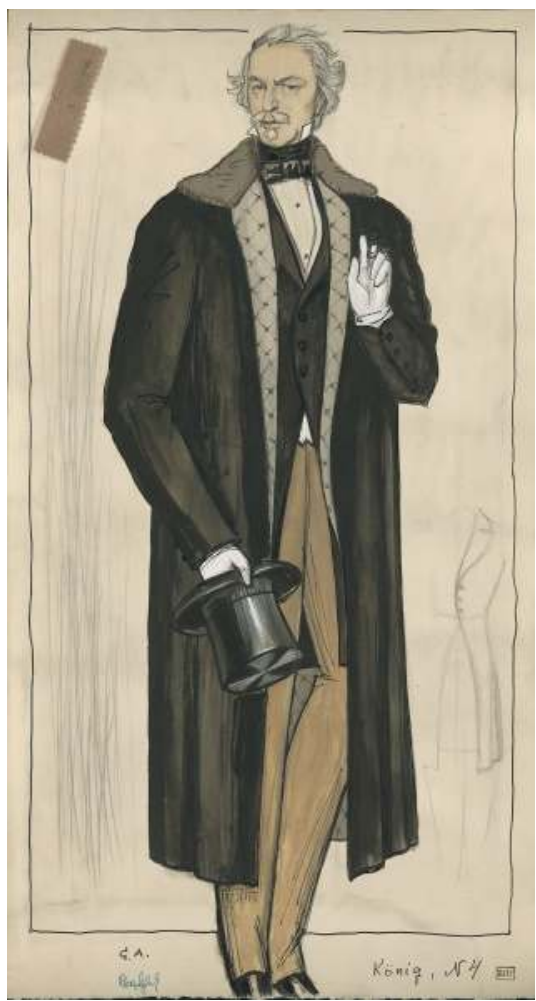

(c) Dominique Rabusson, coll. Cinémathèque française, Paris, D104-059. 
Illustration 22 : Georges Annenkov, Patrie : Lucien Nat - Duc d'Albe, 1945, mine de graphite, gouache sur papier, $50 \times 32,5 \mathrm{~cm}$.

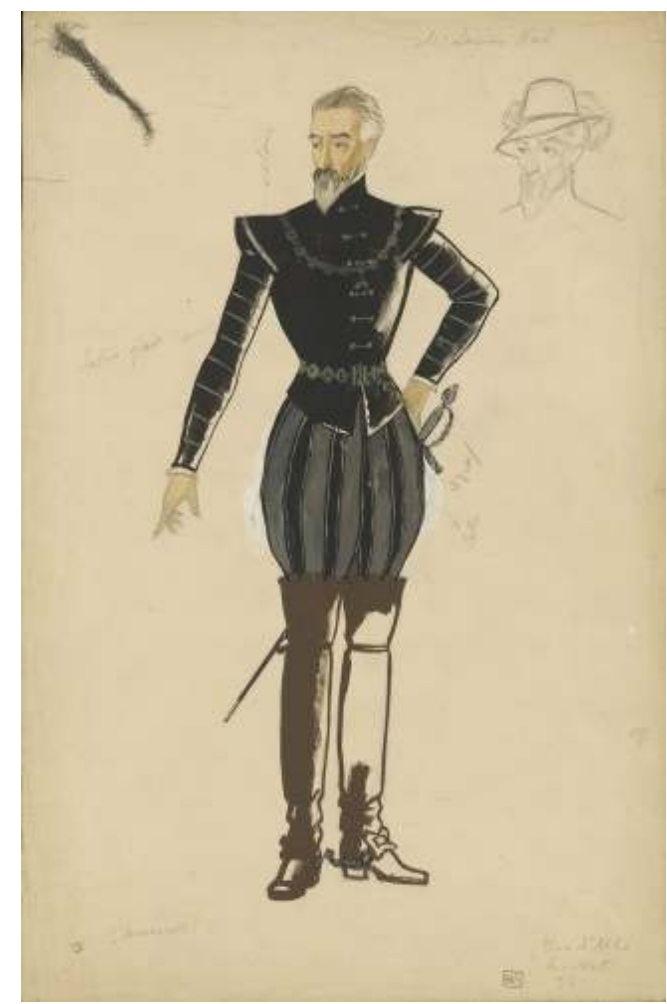

(C) Dominique Rabusson, coll. Cinémathèque française, Paris, D104-050 
Illustration 23 : Rosine Delamare, French Cancan : Nini se rhabille dans le fournil, 1954, mine de graphite, gouache sur papier, $50 \times 3,5 \mathrm{~cm}$.

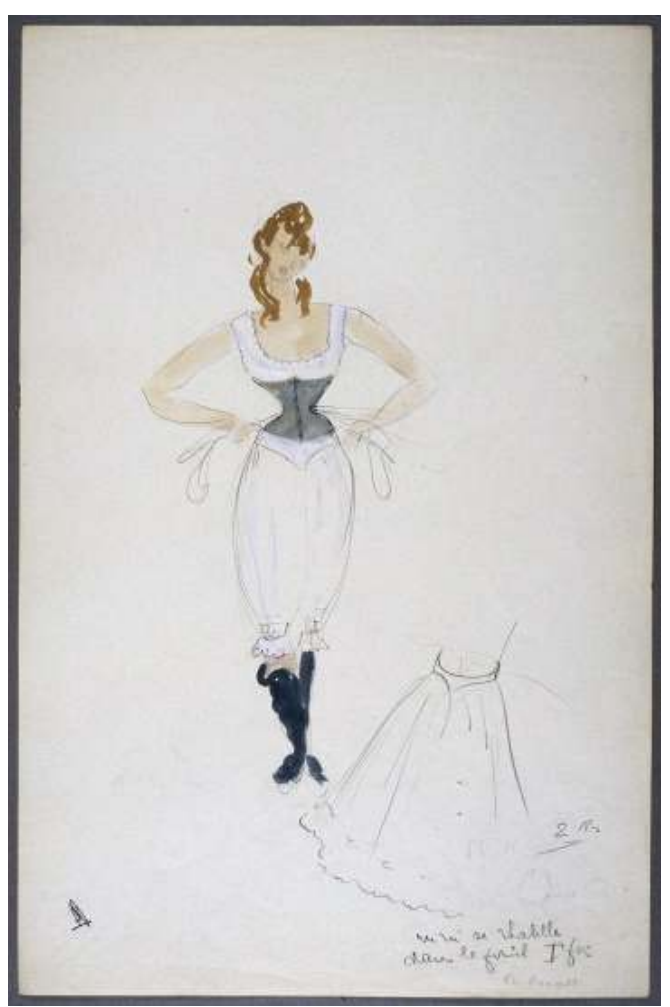

(c) Anne-Marie Spitzer, coll. Cinémathèque française, Paris, D025-038. 
Illustration 24 : Georges Annenkov, Patrie : Maria Mauban - Élisabeth, 1945, mine de graphite, encre, gouache sur papier, $50 \times 32,5 \mathrm{~cm}$.

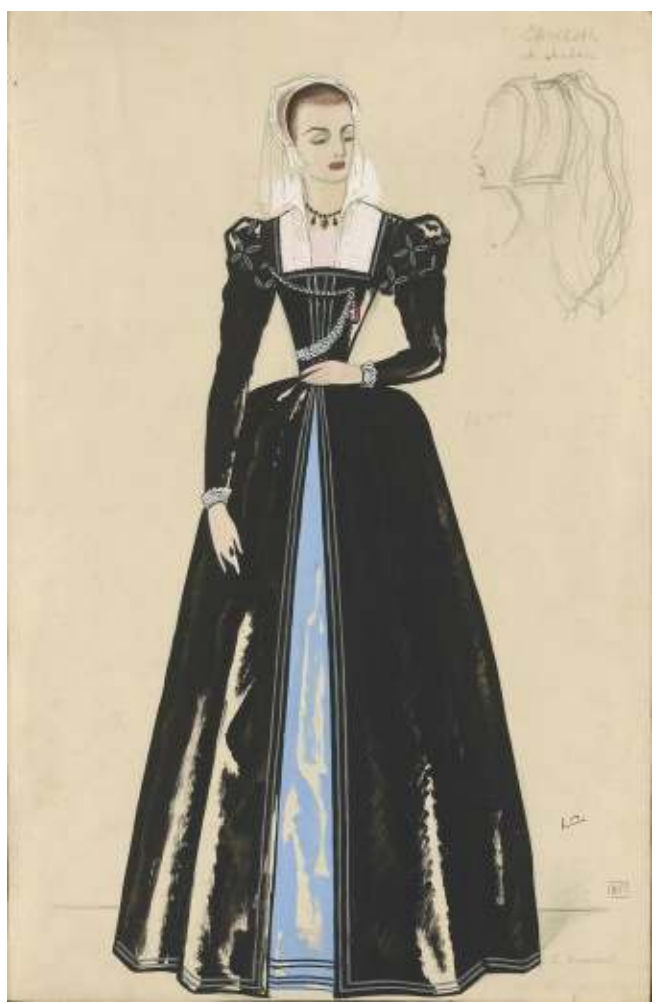

(c) Dominique Rabusson, coll. Cinémathèque française, Paris, D104-051. 
Illustration 25 : Georges Annenkov, L'Affaire du collier de la reine, 1945, mine de graphite, aquarelle, gouache sur papier, $48 \times 31,5 \mathrm{~cm}$.

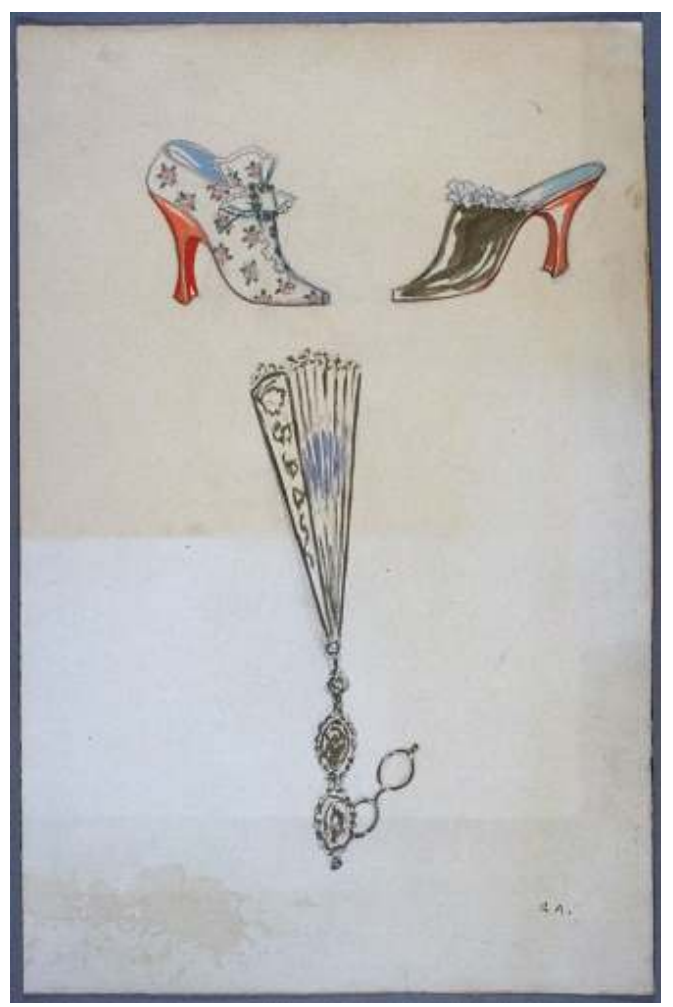

(c) Dominique Rabusson, coll. Cinémathèque française, Paris, D003-067. 
Illustration 26 : Rosine Delamare, Les Grandes Manœuvres, 1955, aquarelle, gouache sur papier, $50 \times 32 \mathrm{~cm}$.

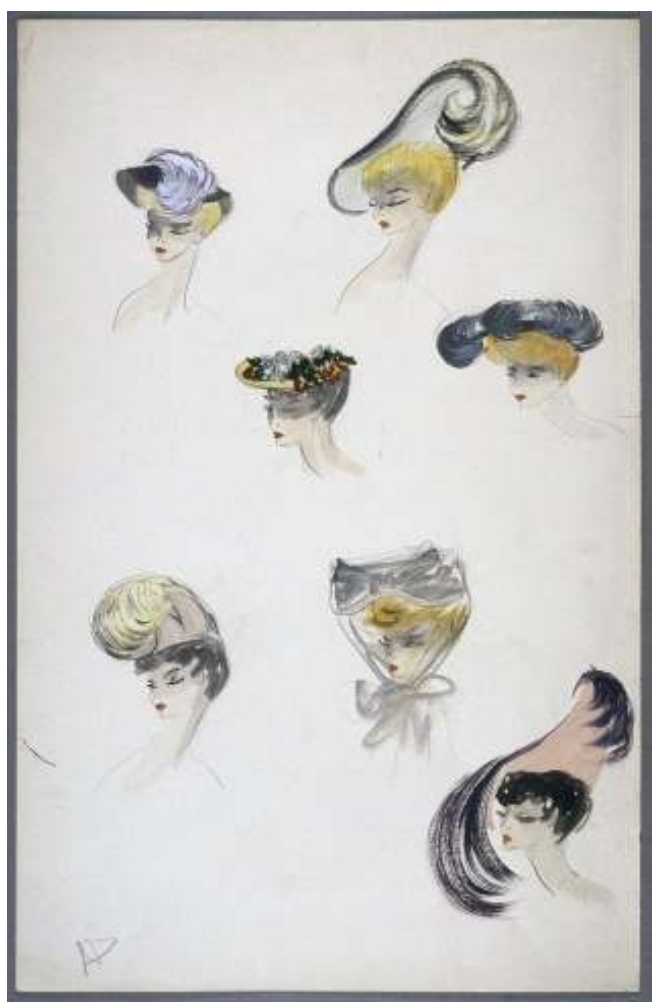

(c) Anne-Marie Spitzer, coll. Cinémathèque française, Paris, D026-006. 
Illustration 27 : Marcel Escoffier, Le Secret de Mayerling, 1948, mine de graphite sur papier, $50 \times 32,5 \mathrm{~cm}$.

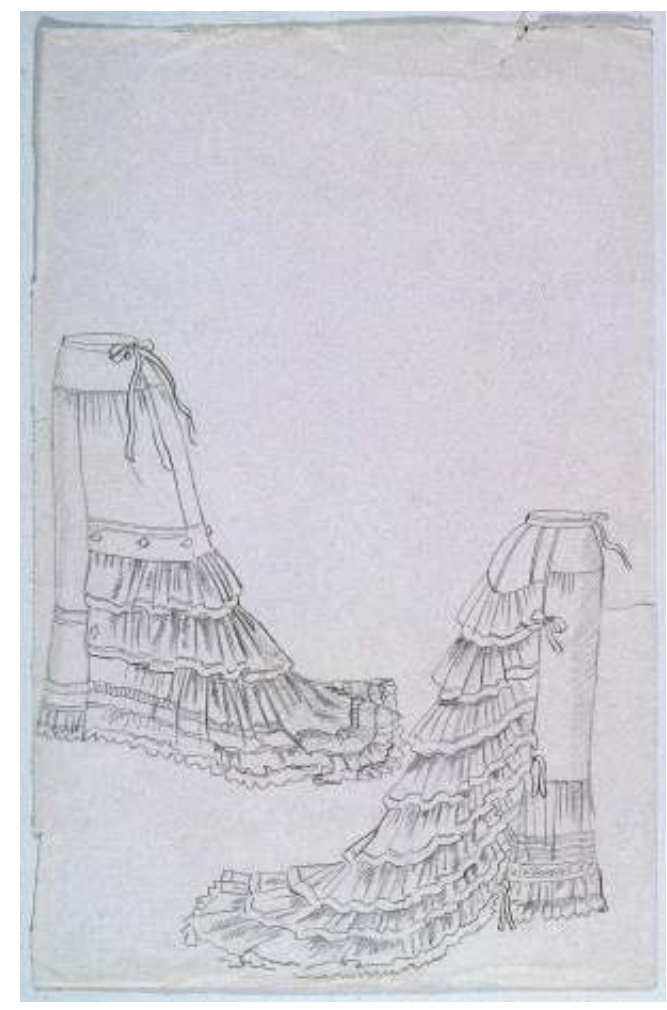

(c) Carol Escoffier, coll. Cinémathèque française, Paris, D102-032.

Le fait que les maquettes soient des représentations utilitaires est évident lorsque l'on observe des divergences sur les habitudes de transcription réaliste des personnages. Des créateurs comme Annenkov tracent des corps «amputés», comme on peut le voir sur les dessins «Julien Sorel n6» (D104-027) et « 7 7 (D104-028) pour Le Rouge et le Noir. Même s'il va jusqu'à l'étape de la mise en couleurs, Annenkov ne prend pas le temps de dessiner tout le corps si cela n'apporte aucune information supplémentaire, omettant ainsi un bras ou une jambe. Les couleurs ne sont parfois présentes que sur une partie des vêtements. L'absence de couleur sur une partie d'un dessin presque entièrement coloré permet de mieux distinguer les détails, comme la boucle et les éperons des bottes du duc d'Albe sur le dessin D104-050 ${ }^{45}$ de Patrie. Avant d'être belle, une maquette de costume doit être claire pour les techniciens chargés de l'exécution.

Si l'on compare certaines maquettes très abouties avec les costumes des scènes correspondantes, on s'aperçoit que des modifications peuvent encore intervenir pendant la phase de réalisation. Par exemple, pour Les Grandes Mancuvres, les costumes des sœurs Duverger recevant Marie-Louise (D0026-00746) divergent par quelques détails : la tenue de Jeanne ${ }^{47}$ ne comporte pas de ras-du-cou noir, mais un collier en or à pendentif, et les nœuds retroussant la sur-jupe sont disposés plus haut et sont plus grands. Juliette ${ }^{48}$ a échangé sa ceinture grise avec la pointe dirigée vers le haut pour une ceinture bleu foncé pointée vers le bas et ornée d'une boucle, sa guimpe a été allégée, mais est surmontée d'un collier de chien vert foncé, et l'ornement en forme d'un fin nœud gris sur son épaule gauche a disparu au bénéfice d'une montre de col. Les coiffures des deux sœurs ont été adaptées par la coiffeuse Jacqueline Juillard et/ou le perruquier Jules Chanteau et semblent ainsi moins volumineuses. Mais il n'est pas rare 
que l'on s'abstienne de tout changement : pour French Cancan, la maquette représentant Nini se rhabillant dans le fournil (D0025-038 ${ }^{49}$ ) a été exécutée à la lettre ${ }^{50}$. Et, au contraire, il arrive que l'on change presque totalement d'idée, à l'image du manteau du soir d'Éléonore pour aller à la Reine Blanche (D0025-00951) qui a été remplacé par une cape courte framboise, sans col froncé blanc ${ }^{52}$.

Illustration 28 : Jeanne Duverger (à gauche), photogramme des Grandes Manœuvres.

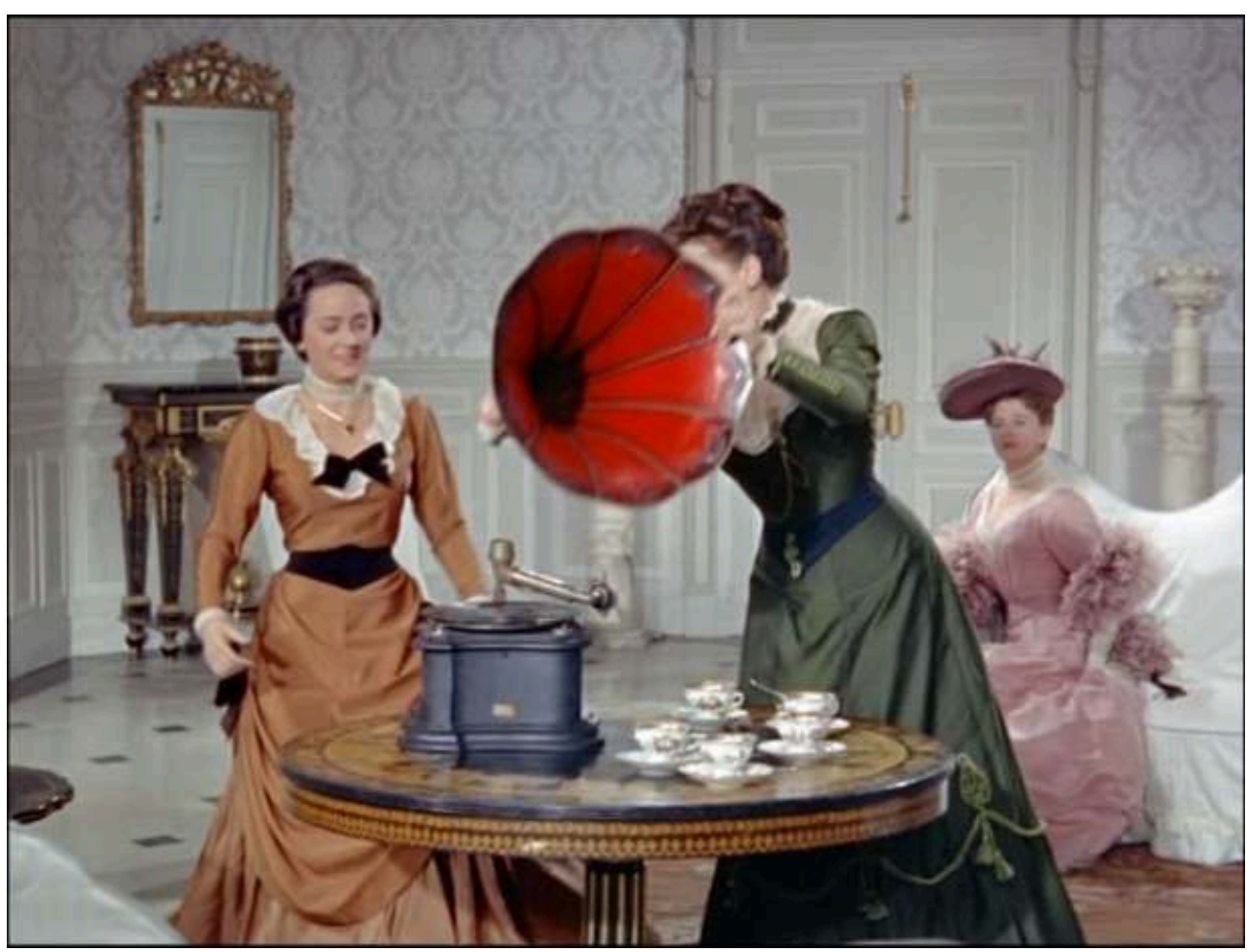


Illustration 29 : Juliette Duverger (à gauche), photogramme des Grandes Manœuvres.

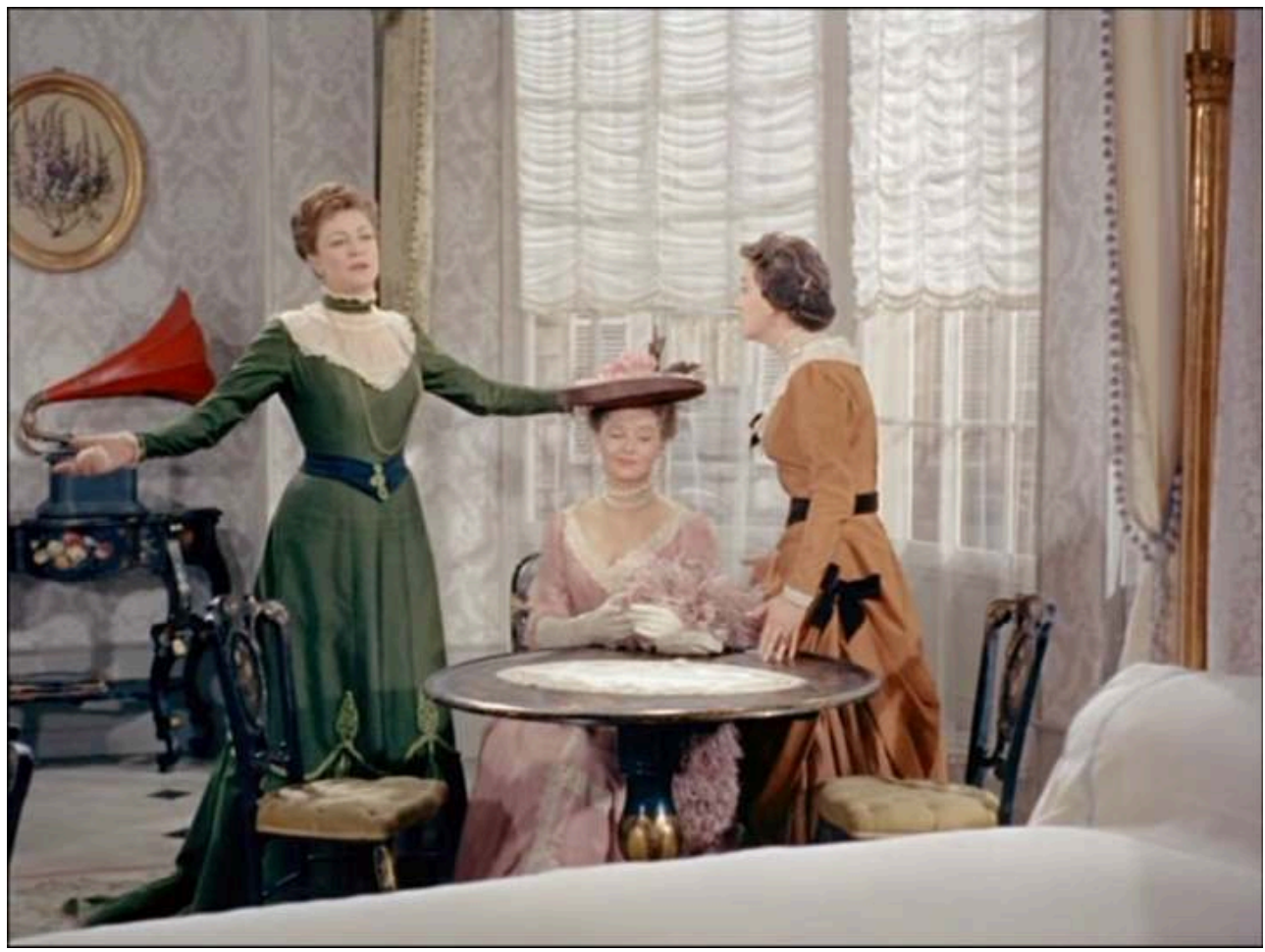

Illustration 30 : Nini, photogramme de French Cancan.

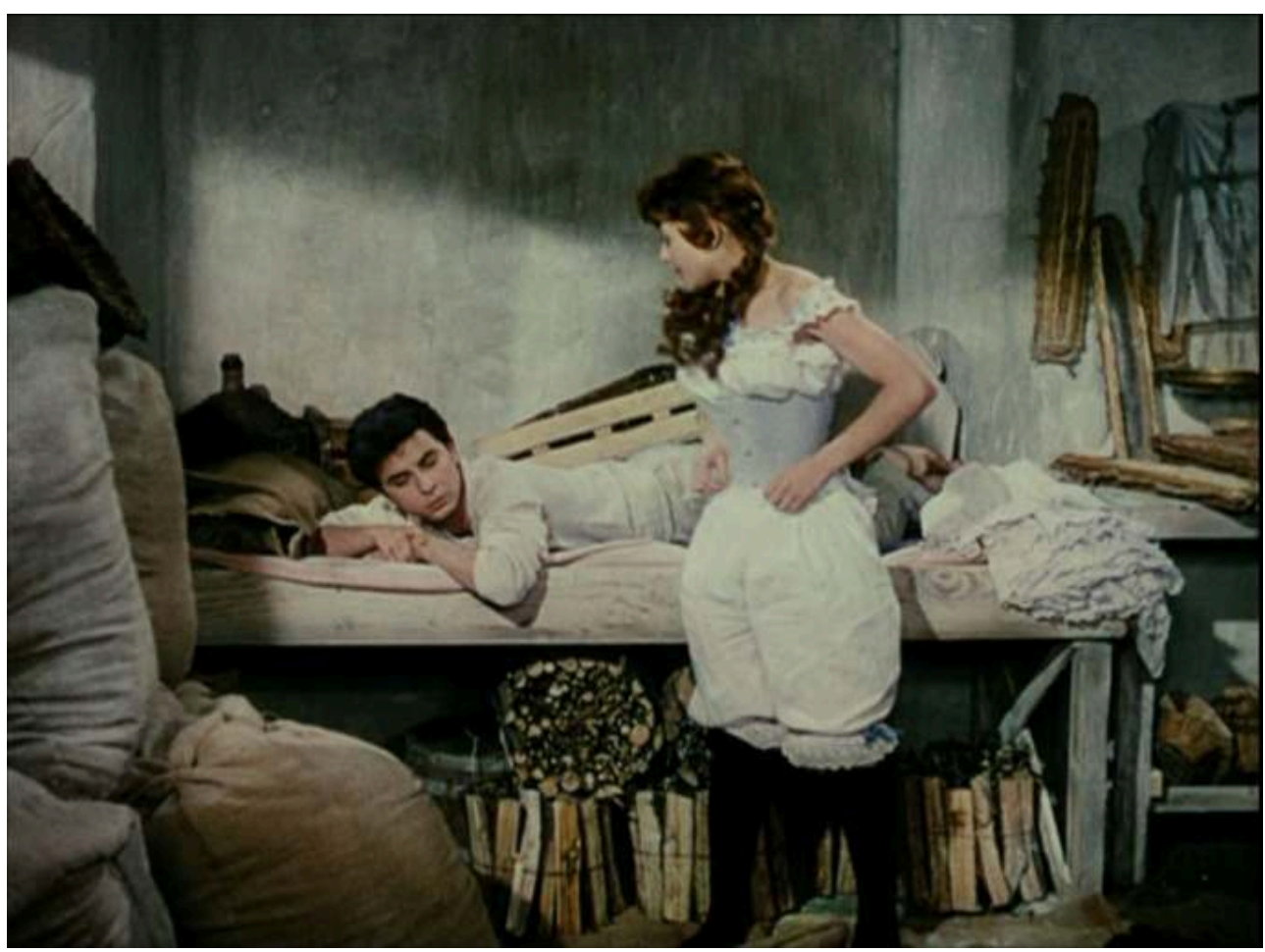


Illustration 31 : Éléonore (à gauche), photogramme de French Cancan.

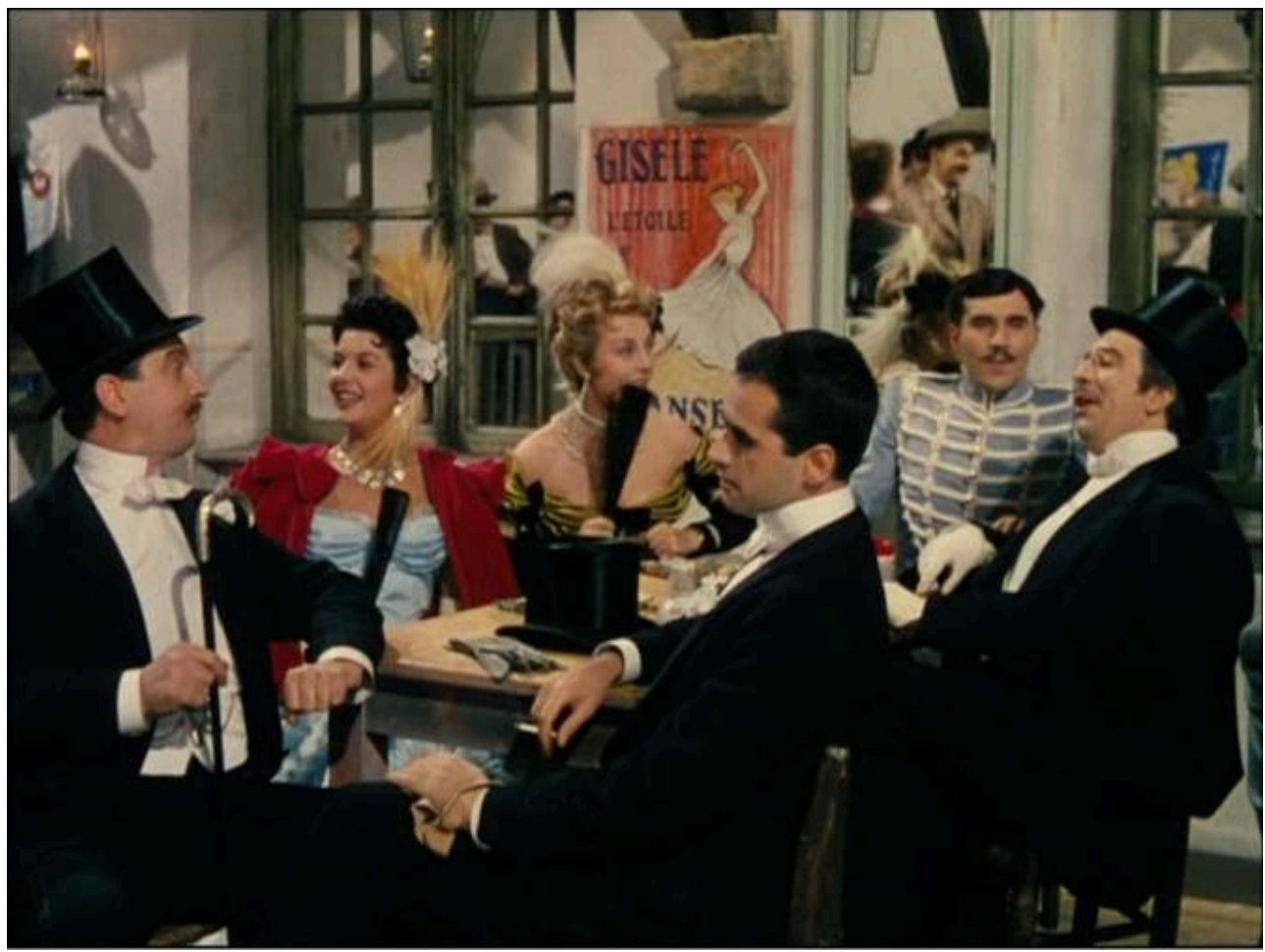

\section{Le rôle des échantillons et des indications manuscrites}

La maquette est un outil de communication qui permet au créateur de costumes de donner une forme peu coûteuse à ses idées et de les présenter à ses interlocuteurs comme le chef décorateur, le directeur de la photographie, le réalisateur, le chef costumier et enfin le costumier coupeur qui l'analyse pour traduire les lignes en volumes textiles. Cependant, le dessin ne suffit pas toujours à remplir cette fonction, la maquette est alors rendue plus tangible grâce à des échantillons de tissu, augmentée d'indications écrites, ou à l'exploitation du verso. Les échantillons sont de petits morceaux de tissus qui font la spécificité des maquettes de costumes et anticipent la phase d'exécution en indiquant des choix de matériaux (et ainsi de propriétés physiques), de couleurs et de motifs. Dans les collections de la Cinémathèque française, les échantillons de tissu sont épinglés ou, beaucoup plus rarement, maintenus par des trombones, comme pour le costume $n^{\circ} 1$ de la baronne Vetsera dans Le Secret de Mayerling (D102-021). Certains tissus sont découpés aux ciseaux cranteurs pour éviter qu'ils s'effilochent, notamment pour le costume $\mathrm{n}^{\circ} 3$ de Mme de Rênal du Rouge et le Noir par Annenkov (D104-032). Ils sont disposés soit au niveau des pièces de vêtement pour lesquels ils seront utilisés, soit les uns à côté des autres avec une inscription spécifiant pour quelle pièce de vêtement ils sont utilisés, à l'image du dessin du costume $\mathrm{n}^{\circ} 11$ de Julien Sorel dans Le Rouge et le Noir (D104-026) qui précise que l'échantillon le plus haut servira à la fabrication des "cols et parements ", le deuxième à la "vareuse » et le dernier au "pantalon ». Il est intéressant de se pencher sur les types de tissu utilisés. Dans le corpus choisi, la laine est préférée pour les classes modestes, notamment pour Les Aventures de Till l'Espiègle, alors que l'on favorise les dentelles, velours ou tissus fins, 
pour les classes aisées, particulièrement les robes de bal, comme la maquette D024-092 des Grandes Manouvres. Des matériaux épais et foncés sont épinglés aux maquettes des tenues de jour des classes aisées, comme le costume $\mathrm{n}^{\circ} 1$ de la baronne Vetsera pour Le Secret de Mayerling (D102-021). Une étude plus poussée des matières textiles permettrait de déterminer si les fibres naturelles étaient privilégiées comme l'ont déclaré plusieurs créateurs de costumes de l'époque qui trouvaient qu'elles reflètent la lumière plus naturellement, permettant d'anticiper le rendu à l'écran. De fait, l'échantillonnage est essentiel, car les propriétés physiques des tissus (la manière dont ils revoient la lumière) déterminent, avec la photographie et le type de pellicule employé, l'aspect des costumes à l'écran.

Les maquettes portent des inscriptions donnant divers types d'informations qui ne sont pas systématiquement positionnées au même endroit sur la page. Ces annotations mentionnent le titre du film, parfois le nom du réalisateur, comme on peut le voir sur la maquette du vendeur de journaux de Lola Montès (D003-094), ou encore la scène concernée. Elles précisent le nom du personnage ou son rôle dans l'intrigue, comme « le prétendant » pour le dessin D082-088, et le nom de l'interprète, telle «Mme Cécile Aubry » dans le rôle d'Aline dans Barbe-Bleue (D021-087). On peut ainsi y déceler des changements d'actrices pressenties pour un rôle, notamment pour celui de Béatrix dans French Cancan, où France Roche remplace Colette Deréal dont le nom a été barré (D025-028). On trouve aussi des informations temporelles : une année et, dans de rares cas, comme celui du «14 octobre » de la maquette de la Belle Abbesse (D046-025), une date précise. Mais il est difficile de déterminer à quel événement correspond ce type de date. Est-ce celle de la prise des mensurations ? Est-ce celle de l'essayage ? Est-ce la date pour laquelle le costume doit être prêt? Est-ce la date de son approbation? Le costume $\mathrm{n}^{\circ} 9$ (ou 5) de la Belle Abbesse porte la même date, alors que le costume $\mathrm{n}^{\circ} 6$ de la scène de la venue du ministre (D025-029) ainsi que le costume ${ }^{\circ} 5$ de la Belle Abbesse pour une scène au Moulin Rouge et dans un fiacre (D025-031) portent la date du « 22 octobre ». Sachant que le tournage s'est déroulé du 4 octobre au 20 décembre 1954, on peut supposer qu'il s'agit des dates pour lesquelles les tenues devaient être achevées. Ainsi, les tenues n'étaient pas toujours terminées avant le tournage.

Les inscriptions précisent le type de vêtement dessiné, par exemple, un "déshabillé » pour L'Amour, Madame (D020-094). Elles désignent aussi le costume, notamment en mentionnant son apparition dans le scénario. Pour L'Amour, Madame, le " costume de départ» (D020-096) s'oppose au «costume d'arrivée» (D020-095). Sur le dessin D024-081 des Grandes Manouvres, l'inscription «Lucile Bal (robe empruntée qu'elle déchire) » précise, de plus, le rôle du vêtement dans l'intrigue. On peut parfois remarquer des changements d'attribution d'un costume d'une scène à une autre. Par exemple, la robe $\mathrm{n}^{\circ} 6$ de la Belle Abbesse dans French Cancan (D025-029) était prévue pour une scène à la Reine Blanche, puis la mention a été barrée et une nouvelle inscription indique qu'elle a été attribuée à la scène de visite du ministre et de la bagarre. La tenue est aussi distinguée par son numéro, par exemple «Mme de Rénal N3 » (D104-032) ou " Julien Sorel - N8 » (D104-029) pour Le Rouge et le Noir. Il arrive que la numérotation change: sur le dessin D025-032, représentant une robe de la Belle Abbesse au Moulin Rouge dans French Cancan, le numéro " 14 » a été barré et remplacé par « 15 ». Parfois, on lit deux numéros sur une même planche, par exemple « 10 » et « 12 » pour le costume de la Belle Abbesse dans une scène au Moulin Rouge (D025-033). On mentionne aussi des vêtements qui complètent le costume, mais ne sont pas 
présents sur la maquette, comme un «manteau » porté par Éléonore dans la scène du Paravent chinois de French Cancan (D025-004). Les pièces de certaines tenues ont été recombinées avec d'autres pièces pour créer de nouvelles tenues pour d'autres scènes. Par exemple, sur la maquette du costume de la colonelle lors de la scène de préparation du bal de la Croix-Rouge dans Les Grandes Mancuvres est précisé : «+ chez elle avec un autre corsage ». Ce remploi de la jupe dans une autre scène montre une tendance à l'économie lorsque c'est possible.

Les inscriptions mettent aussi en avant la dimension collective du travail du costume. Les maquettes comportent parfois une marque d'approbation de la part du réalisateur, comme la signature de Max Ophuls sur le dessin représentant le costume «n1» de "Ludwig der Erste» (D104-060) de Lola Montès. La présence de deux maquettes de costumes présumées dessinées par le chef décorateur Max Douy ${ }^{53}$, pour La Jument verte de Claude Autant-Lara (1959, Eastmancolor), film dont les costumes ont été conçus par Rosine Delamare, pose question. Il semblerait que dans de rares exceptions la conception de certaines tenues pouvait être confiée à un technicien autre que le créateur de costumes. Dans le cas présent, la raison plausible est qu'il s'agissait de créer un costume " animalier ", ce qui correspondait peut-être plus aux compétences de Max Douy qu'à celles de Rosine Delamare. Cela montre l'aspect collaboratif et pas totalement fermé du travail du costume dans le cinéma français d'après-guerre.

On découvre aussi des informations relatives aux questions pratiques d'exécution des costumes. Les créateurs indiquent les textiles constituant le costume, par exemple "satin et tulle ", "bordure de dentelle " pour un déshabillé dessiné par Blanche van Parys pour L'Amour, Madame (D020-094). Certains précisent l'effet des tissus choisis : sur le dessin D102-036, représentant Mme Duveyrier après son deuil dans Pot-Bouille, Marcel Escoffier spécifie : «tous les tissus de même valeur - seules les différences de matière jouent». Encore une fois, les hésitations sont visibles: sur la maquette représentant la Belle Otéro dans le film de Richard Pottier (D102-041), Escoffier a d'abord écrit "mousseline ou velours ou faille " pour le textile principal, démontrant une hésitation dans le choix des matières et ainsi, chacune ayant des propriétés physiques bien distinctes, dans l'aspect et le tombé de la robe. Puis Escoffier a pris une décision en barrant «mousseline » et "faille » et en ajoutant "vert nacré ». On peut observer sur les maquettes des suites de chiffres qui détaillent certainement les mensurations des interprètes. On déchiffre sur la maquette du costume de Thérèse dans French Cancan (D025-022) les indications suivantes, regroupées par trois et réparties sur trois lignes superposées : 85-54-88/45-155-38/14-46-74. Cependant, il est difficile de savoir à quel type de mesure correspond chaque chiffre. On peut supposer que les trois premiers numéros correspondent au tour de poitrine, tour de taille et tour de hanches de l'actrice Annick Morice et que la hauteur entre le sol et ses épaules est de $155 \mathrm{~cm}$. Le « 14 » pourrait correspondre à son tour de poignet et le « 45 » à la largeur de ses épaules. Escoffier indique que l'ornement des épaules du costume $\mathrm{n}^{\circ} 12$ de Maria Vetsera dans Le Secret de Mayerling (D102-029), composé d'une rose et de dentelle, serait «trop lourd». Aujourd'hui, il est impossible de savoir s'il faut comprendre que la garniture serait visuellement surchargée ou physiquement trop lourde. Avec la coiffure de Lucrèce Borgia (D102-054), Escoffier précise la mise en œuvre de sa création. Il explique comment composer la coiffure: "Voile transparent bordé de perles et recouvrant l'entière coiffure. Sur les deux côtés: rouleaux de tresses. »Certaines inscriptions montrent que les maquettistes prévoient de fabriquer seulement une ou deux pièces d'une tenue. C'est ce que suggère la maquette D024-089 ${ }^{54}$, représentant 
Marie-Louise Rivière dans Les Grandes Manœuvres : la tenue est entièrement dessinée, mais seuls les vêtements au-dessus de la taille sont mis en couleurs, et une inscription précise « jaquette pour porter sur jupe grise », ce qui laisse penser que Rosine Delamare compte réutiliser une jupe grise d'une autre tenue ou se procurer une jupe grise sans la faire fabriquer, peut-être en la louant. Cependant, des instructions restent énigmatiques, comme « 12 [?] par costume fait neuf pour le film complet » concernant les mercenaires des Aventures de Till l'Espiègle (D024-026).

Illustration 32 : Rosine Delamare, Les Grandes Manœuvres : Michèle Morgan, 1955, mine de graphite, gouache sur papier, $50 \times 32 \mathrm{~cm}$.

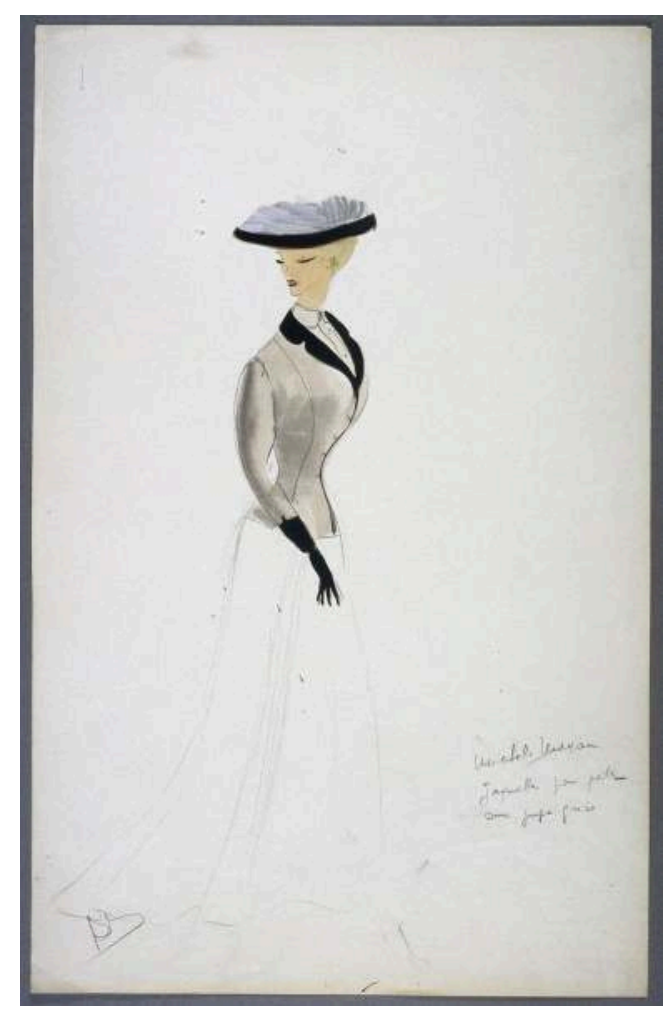

(c) Anne-Marie Spitzer, coll. Cinémathèque française, Paris, D024-089.

Pour un chercheur, la page sur laquelle est décrit le costume n'est pas la seule intéressante. L'étude du verso des maquettes est également riche de renseignements sur la création des costumes. Rosine Delamare a utilisé le revers du dessin des officiers espagnols des Aventures de Till l'Espiègle (D024-033) pour faire des essais, s'entraîner à tracer les hauts-de-chausses et pourpoints bouffants des soldats. Les créateurs exploitent le revers de leur feuille pour compléter la représentation principale. Rosine Delamare a ainsi dessiné au verso de la maquette D024-031 des Aventures de Till l'Espiègle le même costume, mais avec un autre point de vue : le dos de la cape de Nèle, laquelle est déjà figurée de face au recto. Au revers de la Belle Abbesse au Paravent chinois dans French Cancan (D010-020), on trouve une variante esquissée du costume. Sur cette version, la jupe de la robe est en deux parties : une englobant les fesses et les genoux et une autre plissée allant jusqu'au sol. Au revers de la maquette du costume $\mathrm{n}^{\circ} 1$ de Louis $\mathrm{I}^{\text {er }}$ de Bavière dans Lola Montès (D104-060), se trouve un papier collé présentant un schéma de l'épaulette du dessin principal avec des précisions détaillant sa composition, notamment "bouton", "cuivre», "noir", "1 doigt». Sur plusieurs maquettes conçues par Rosine Delamare, comme celle d'une robe de bal pour Les Grandes 
Manœuvres (D024-092), se trouve le prénom «Georgette». Il s'agit de celui de son assistante Georgette Fillon. À l'image des rectos, les versos nous informent sur la phase de fabrication des costumes. Le dos du dessin de jupon du Secret de Mayerling $\left(D 102-032^{55}\right)$ comporte le tracé du contour d'un pied et l'inscription « $20 \mathrm{~cm}$ », qui correspond à l'évidence à la taille de ce pied. Ces éléments ont certainement servi à fabriquer la paire de chaussures qui accompagnait la tenue dont le jupon est représenté à l'avers. La maquette d'un costume de Brigitte Bardot pour Les Grandes Mancuvres (D024-079) porte des inscriptions en anglais derrière les échantillons de tissu: «biscuit » et "same green ». Il s'agit peut-être d'instructions invitant les couturières à respecter les couleurs désignées lors d'une fabrication à l'étranger, dans un pays anglophone. Sur la maquette D102-037 de Nana, l'inscription "Bozzetti di costumi», signifiant "ébauche de costume", incite à penser que les costumes du film ont été fabriqués dans un atelier italien; mais Escoffier préférait peut-être créer en italien (langue qu'il parlait). Une inscription, peut-être postérieure à la production du film, sur la maquette du costume de Thérèse dans Les Grandes Manœuvres (D024-091) indique que ce vêtement reste « non réalisé ». Ainsi, comme l'explique Annenkov, après l'exécution, les maquettes «reviendront, martyrisées, souillées, flétries, couvertes de notes, au crayon ou à l'encre, et de signatures intruses, dans les cartons de leur auteur pour s'ajouter à la documentation de sa bibliothèque et, peut-être, un jour tomberont-elles entre les mains de futurs archivistes des cinémathèques ${ }^{56}$ ».

Les maquettes de costumes du cinéma français d'après-guerre sont des œuvres qui paradoxalement n'ont pas vocation à être directement vues du public, ne sont conçues que pour servir une autre œuvre: le film ${ }^{57}$. La qualité esthétique de ces créations, traduisant graphiquement le caractère vivant des étoffes avant leur réelle mise en mouvement dans le film, révèle les styles personnels d'artistes aux méthodes créatives variables. Par les choix qu'elles marquent, les maquettes révèlent une part des intentions esthétiques des films. Elles démontrent aussi que ces choix n'étaient pas des évidences, mais résultaient d'un processus fait d'hésitations et d'abandons. Les maquettes de costumes servent un film en donnant une première forme visible, temporaire et économique, à des idées de costumes afin qu'elles soient discutées et approuvées par l'équipe puis exécutées. Leur rôle de messager façonne leurs spécificités: les échantillons de tissu rendent plus concret le projet et les mots précisent les enjeux techniques et esthétiques. Outils d'un travail artistique, les maquettes de costumes renseignent les chercheurs d'aujourd'hui sur les méthodes professionnelles des créateurs de costumes de cinéma dans l'après-guerre.

\section{NOTES}

1. Les pellicules captant différemment la lumière suivant leur émulsion, le rendu de l'image lors de la projection s'éloigne de la réalité, et ce, de manière particulièrement évidente avec le noir et blanc.

2. Pierre Étaix était assistant réalisateur alors que Jacques Cottin, lui aussi collaborateur récurrent de Jacques Tati, est crédité à la " régie costumes " au générique de Mon oncle. Dans 
Étaix dessine Tati: portrait d'une collaboration (ACR, 2007), Francis Ramirez et Christian Rolot expliquent que Pierre Étaix imaginait les tenues des personnages. Cependant, au sein de la donation de la Cinémathèque française, il est difficile de catégoriser certains dessins (gag, costume, décor...) et de savoir si les représentations de personnages ont été directement utilisées comme maquettes (sans annotation ni échantillon) de costumes dont l'exécution a été dirigée par Jacques Cottin, si elles ont servi de modèles à Jacques Cottin lorsqu'il a conçu les costumes ou si Pierre Étaix a déposé sur le papier les fruits d'une réflexion commune avec Jacques Cottin. On peut simplement noter que certains costumes apparaissant à l'écran sont très fidèles aux dessins correspondants de Pierre Étaix.

3. Peintre d'origine russe naturalisé français, formé à l'École des beaux-arts de SaintPétersbourg, Georges Annenkov (1889-1974) était un chef décorateur et créateur de costumes œuvrant principalement pour le cinéma mais aussi pour les arts de la scène. Dès les années 1910, il a voyagé en France avant de s'y installer définitivement en 1924, ce qui lui donnera l'occasion de travailler avec Anatole Litvak, Abel Gance, Jacques de Baroncelli, Christian-Jaque, Jean Delannoy ou Carmine Gallone. Écrivain, il raconte sa collaboration avec le réalisateur de Madame de... et Lola Montès dans Max Ophüls (Le Terrain vague, 1962), ainsi que ses souvenirs et sa conception de la création de costumes dans En habillant les vedettes (Robert Marin, 1951).

4. Illustration 1.

5. Illustration 1.

6. Illustration 2.

7. Georges Annenkov, En habillant les vedettes, Paris, Robert Marin, 1951, p. 305.

8. Rosine Delamare (1911-2013) est une créatrice de costumes française, formée au dessin à Paris, ayant travaillé de 1939 à 1983 sur plus de cent-dix films principalement français mais aussi américains. Julien Duvivier est le premier à lui donner sa chance avant Claude Autant-Lara, Christian-Jaque, André Cayatte, Henri Verneuil, René Clair, Jean Renoir, ou Jean Delannoy. Elle a aussi œuvré pour les arts de la scène et la télévision.

9. Marcel Escoffier (1910-2001) a étudié à l'École des arts décoratifs à Paris et commencé sa carrière au cinéma en tant qu'assistant décorateur auprès de Christian Bérard. Entre 1938 et 1987, ce créateur de costumes français, très apprécié de l'actrice Martine Carol, a fréquemment collaboré avec Jean Cocteau, Christian-Jaque ou Jean Delannoy. Il a aussi œuvré pour le cinéma italien, notamment pour Luchino Visconti et Vittorio De Sica, et pour les arts de la scène.

10. Georges Koskas (1922-2013), né en Tunisie et formé dans les ateliers de Michel Seuphor, André Lhote et Fernand Léger après la seconde guerre mondiale, est un peintre, illustrateur et poète français rattaché à l'abstraction géométrique. Dans les années 1950, il s'approche de l'art informel avant de se tourner vers la figuration, les meubles peints, la photo-peinture ainsi que le roman-photo. Ancien étudiant de l'Idhec, il a travaillé comme décorateur et créateur de costumes pour la télévision et le cinéma, notamment pour La Poupée de Jacques Baratier en 1962.

11. Antoine Mayo (1905-1990), né en Égypte, gagne Paris en 1923 pour étudier l'architecture, mais préfère passer son temps avec les artistes de Montparnasse et entre finalement à l'École des beaux-arts. Proche du mouvement surréaliste, le peintre se lie d'amitié avec Jacques Prévert. Mayo crée ses premiers costumes de cinéma pour Les Enfants du paradis en 1943 puis ceux de onze autres films de Marcel Carné jusqu'en 1965. Il conçoit des tenues pour les productions d'autres grands réalisateurs français de l'après-guerre comme René Clair, Jacques Becker, Alexandre Astruc ou René Clément. Il œuvre aussi pour le cinéma hollywoodien avec La Terre des pharaons (Howard Hawks, 1954) et exerce en parallèle pour les arts de la scène et comme décorateur.

12. Illustration 3 .

13. On sait peu de choses sur la carrière de Blanca de Selva (1916-1986), peintre et illustratrice qui a créé les costumes de plusieurs films, dont Maxime d'Henri Verneuil en compagnie de Rosine Delamare. Elle change de nom en épousant le compositeur Georges van Parys en 1936.

Images du travail, travail des images, 10 | 2021 
14. Georges Wakhevitch (1907-1984), né à Odessa, immigre en France en 1921. Il suit des études d'art et d'architecture à Paris, notamment auprès de Pavel Tchelitchev et à l'École nationale supérieure des arts décoratifs. Il est d'abord comédien et assistant avant de devenir chef décorateur et créateur de costumes pour le théâtre, l'opéra, le ballet, le cinéma et la télévision, jusqu'aux années 1980. Il travaille principalement pour des productions françaises, notamment avec Jean Renoir, Julien Duvivier, Jacques de Baroncelli et Yves Allégret, mais il se met aussi au service du cinéma américain. Il a publié L'Envers des décors (Laffont, 1977).

15. Georges Annenkov, op. cit., 1951, p. 305.

16. Illustration 4.

17. Illustration 5 .

18. Illustration 6 .

19. Illustration 7 .

20. Illustration 8 .

21. Illustration 9.

22. Illustration 10 .

23. Illustration 11.

24. Illustration 1.

25. Illustration 12.

26. Illustration 13.

27. Illustration 3.

28. Illustration 14.

29. Illustration 15.

30. Illustration 16.

31. Illustration 17.

32. Illustration 18.

33. Illustration 19.

34. Illustration 20.

35. Illustration 20.

36. Madeleine Delpierre, Marianne de Fleury, Dominique Lebrun, L'Élégance française au cinéma, Paris, Paris Musées et Société de l'histoire du costume, 1988, p. 186.

37. Illustration 21.

38. Illustration 22 .

39. Illustration 23.

40. Georges Annenkov, En habillant les vedettes, Robert Marin, Paris, 1951, p. 310.

41. Illustration 24 .

42. Illustration 25.

43. Illustration 26.

44. Illustration 27.

45. Illustration 22 .

46. Illustration 20.

47. Illustration 28.

48. Illustration 29.

49. Illustration 23.

50. Illustration 30 .

51. Illustration 18.

52. Illustration 31.

53. Max Douy (1914-2007), chef décorateur français, a étudié l'architecture et a commencé sa carrière au cinéma dès 1930 comme assistant de Lucien Aguettand, André Andrejew ou Eugène Lourié. C'est en 1942 avec Premier Atout (Jacques Becker) qu'il devient chef décorateur. Il a été récompensé pour son travail dans Occupe-toi d'Amélie (Claude Autant-Lara, 1949) et Le Malevile 
(Christian de Chalonge, 1980). Il a aussi œuvré pour le théâtre et la télévision. Il est l'auteur avec son frère le décorateur Jacques Douy de Décors de cinéma : les studios français de Méliès à nos jours (Le Collectionneur, 1993).

54. Illustration 32.

55. Illustration 27.

56. Georges Annenkov, op. cit., p. 310.

57. C'est une des différences majeures d'avec les dessins de mode : dans la haute couture, l'œuvre finale est le vêtement lui-même.

\section{RÉSUMÉS}

À partir d'un corpus de cent quatre-vingt-treize maquettes de costumes issues de la préparation de vingt-cinq longs métrages de fiction produits en France entre 1945 et 1959, dessinées par huit créateurs de costumes et conservées à la Cinémathèque française, cet article étudie la place de ces œuvres dans le processus de création cinématographique d'après-guerre. La maquette était un objet esthétique et utilitaire indispensable à la création de costumes originaux pour une œuvre filmique singulière au sein d'un art collectif. Elle est généralement constituée de trois éléments : l'indispensable dessin qui révèle le style personnel de chaque créateur et a pour rôle de rendre visibles leurs idées ; des échantillons de tissus indiquant quelles matières et teintes ont été choisies pour la fabrication des pièces, les rendant ainsi plus tangibles; et des inscriptions donnant des précisions aux collaborateurs pour l'exécution et le tournage.

With a corpus of one hundred and ninety-three costumes sketches from the preproduction of twenty-five fiction feature films produced in France between 1945 and 1959, drawn by eight costume designers and kept at Cinémathèque française, this article studies the part of these works in the process of filmmaking after the Second World War. The sketches were aesthetic and practical objects essential to the creation of original costumes for a particular film within a collective art form. Sketches were generally made up of three elements: the essential drawing which reveals the personal style of each designer and has the role of making visible their ideas; fabric samples indicating which materials and colours were chosen for the manufacture of the pieces, making them more tangible; and inscriptions giving details to collaborators for execution and shooting.

\section{INDEX}

Mots-clés : costume, maquette, dessin, cinéma, coiffure, créateur de costumes

Keywords : Costume design, costume, hairstyle, sketch, film studies

\section{AUTEUR}

\section{MYRIAM FOUILLET}

IRCAV, Université Sorbonne Nouvelle-Paris 3. Après un Master d'études cinématographiques à l'université Paris 7 et un premier cycle d'histoire de l'art à l'école du Louvre en spécialité 
" Histoire de la mode et du costume », Myriam Fouillet prépare une thèse, avec le soutien de la Cinémathèque française, sur le processus de création des costumes pour le cinéma français entre 1945 et 1959 sous la direction de François Thomas à la Sorbonne Nouvelle, université où elle a coorganisé un colloque sur le costume dans les arts du spectacle (2019). Elle enseigne à Rennes 2 l'analyse des costumes. 